\title{
Nonlinear Schrödinger Equations with Steep Magnetic Well
}

\author{
Shin-ichi SHIRAI \\ Osaka Institute of Technology \\ (Communicated by K. Shinoda)
}

\begin{abstract}
We study the nonlinear magnetic Schrödinger equation, $-(\nabla-i \lambda A)^{2} u=f\left(x,|u|^{2}\right) u$ on $\mathbf{R}^{N}$, where $N \geq 2$ and the nonlinearity is super-linear and subcritical. The vector potential $A$ and the associated magnetic field are assumed to vanish on a common bounded open set $\Omega$. It is shown that the equation above has more and more solutions which are localized near $\Omega$ as $\lambda \rightarrow \infty$.
\end{abstract}

\section{Introduction and results}

In this paper, we study the nonlinear Schrödinger equation with magnetic field,

$$
-(\nabla-i \lambda A(x))^{2} u(x)=f\left(x,|u(x)|^{2}\right) u(x)
$$

on $\mathbf{R}^{N}$, where $\lambda>0$ is a large parameter and $N \geq 2$. We are concerned with the existence of multiple solutions which are localized near the interior of the zero set of the magnetic potential as $\lambda \rightarrow \infty$.

This kind of the "steep-potential-well" problem for equations of the form $-\Delta u+$ $(\lambda V(x)+W(x)) u=f(x, u)$ as $\lambda \rightarrow \infty$, where the interior of the zero set $V^{-1}(0)$ is assumed to be nonempty, has been studied by many authors under various conditions on the potentials and the nonlinearity (See, e.g., [Ba-Wa], [Ba-Wa2], [Ba-Pa-Wa], [Si], [De-Di], [Cl-Di], [Di-Ta], [Al], [Al-So], [Al-Mo-So], and the references therein). In [Ta1], [Ta2], and [Ta3], Tang considers the corresponding problem for the equation with magnetic field, $-(\nabla-i A(x))^{2} u+(\lambda V(x)+W(x)) u=f(u)$.

Our result gives a magnetic analog of these works, replaced the steep scalar potential well by the steep magnetic well. The proof is based on the argument used in [Ba-Pa-Wa] and [Ba-Wa2].

We fix some notation. By " $A+B=: C+D$ ", we mean that $C$ and $D$ are defined by $A$ and $B$, respectively. We denote by $|\cdot|$ the standard Euclidean norm. For any metric space

Received March 30, 2009; revised March 14, 2012; revised November 27, 2012 2000 Mathematics Subject Classification: 35J60, 35B20, 35J10

Key words and phrases: NLS, steep magnetic well, variational methods, existence of multiple solutions, concentration to the Dirichlet problem

This work is partially supported by JSPS KAKENHI 22740095, Grant-in-Aid for Young Scientists (B). 
$M$, we set $B_{r}(x)=\{y \in M \mid \operatorname{dist}(x, y) \leq r\}$. For any subset $S$ of $M$, we set $\operatorname{dist}(x, S)=$ $\inf _{y \in S} \operatorname{dist}(x, y)$ and set $S_{\delta}^{+}=\{x \in M \mid \operatorname{dist}(x, S) \leq \delta\}$ for $\delta>0$.

For any subset $\Omega$ of a topological space $X$, we denote the closure, the complement, the interior, and the boundary of $\Omega$ by $\bar{\Omega}, \Omega^{c}$ (or $X \backslash \Omega$ ), int $(\Omega)$, and $\partial \Omega$, respectively.

We denote by $F\left(M, M^{\prime}\right)$ the space of all $M^{\prime}$-valued $F$-functions on $M$, and we denote $F\left(\mathbf{R}^{N}, \mathbf{C}\right)$ by $F\left(\mathbf{R}^{N}\right)$. We denote by $C_{0}^{\infty}\left(\mathbf{R}^{N}\right)$ the space of all (complex-valued) smooth functions on $\mathbf{R}^{N}$ with compact support. We say that $u_{n} \rightarrow u$ in $L_{\mathrm{loc}}^{q}\left(\mathbf{R}^{N}\right)$ if $u_{n} \rightarrow u$ in $L^{q}(K)$ for each compact subset $K$ of $\mathbf{R}^{N}$.

The symbol $2^{*}$ stands for $2 N /(N-2)$ if $N \geq 3$ and for $\infty$ if $N=2$. For any sequence $\left\{u_{n}\right\}_{n}$ and an element $u$ in a Banach space $X$, we write " $u_{n} \rightarrow u$ " if the sequence $\left\{u_{n}\right\}_{n}$ converges weakly to $u$ in $X$ as $n \rightarrow \infty$. The notation $X^{\prime}\langle\cdot, \cdot\rangle_{X}$ stands for the coupling between the dual $X^{\prime}$ and $X$.

By abuse of notation, we write, e.g., $f\left(x,|u|^{2}\right)$ to indicate either the function $f\left(\cdot,|u|^{2}(\cdot)\right)$ or the value $f\left(x,|u(x)|^{2}\right)$ if there is no fear of confusion.

For a magnetic vector potential $A=\left(A_{1}, \ldots, A_{N}\right)$, we define the magnetic field $B=$ $d A=\left(B_{j k}\right)_{j, k=1}^{N}$, where $B_{j k}=\partial_{j} A_{k}-\partial_{k} A_{j}$. Define

$$
|B(x)|=\left(\sum_{j<k}\left|B_{j k}(x)\right|^{2}\right)^{1 / 2}
$$

and

$$
\gamma_{B}(x)=\left(\sum_{j<k}\left|\nabla\left(\frac{B_{j k}(x)}{|B(x)|}\right)\right|^{2}\right)^{1 / 2} .
$$

To formulate the main results, we make the following assumptions.

(A.1) The magnetic vector potential $A$ belongs to $C^{2}\left(\mathbf{R}^{N}, \mathbf{R}^{N}\right)$.

(A.2) $\liminf |x| \rightarrow \infty|B(x)|>0$. In particular, $B^{-1}(0)$ is bounded.

(A.3) $\lim \sup _{|x| \rightarrow \infty} \gamma_{B}(x)<\infty$.

(A.4) The set $\Omega=\operatorname{int}\left(B^{-1}(0)\right)$ is non-empty and has smooth boundary.

(A.5) $\Omega \subset A^{-1}(0)$ and Meas $\left(B^{-1}(0) \backslash \Omega\right)=0$. Here, Meas $(\cdot)$ stands for the Lebesgue measure.

For the nonlinearity, we make the following assumptions.

(f.1) The function $f$ belongs to $C\left(\mathbf{R}^{N} \times[0, \infty)\right.$, R).

(f.2) There exists $p \in\left(2,2^{*}\right)$ and $C>0$ such that $\sup _{x \in \mathbf{R}^{N}}\left|f\left(x, t^{2}\right)\right| \leq C\left(1+t^{p-2}\right)$ for any $t>0$. Moreover, $\lim _{t \rightarrow+0} \sup _{x \in \mathbf{R}^{N}}|f(x, t)|=0$.

(f.3) There exists $\theta>2$ such that $0<\theta F(x, t) \leq f(x, t) t$ for all $x \in \mathbf{R}^{N}$ and $t>0$, where $F(x, t)=\frac{1}{2} \int_{0}^{t} f(x, \tau) d \tau$.

(f.4) There exists $t_{0}>0$ such that $\inf _{x \in \mathbf{R}^{N}} F\left(x, t_{0}^{2}\right)>0$. 
(f.5) The function $f(x, \cdot)$ is strictly increasing for a.e. $x \in \mathbf{R}^{N}$.

(f.6) There exists $C>0$ such that $\sup _{x \in \mathbf{R}^{N}}\left|f\left(x,|u+v|^{2}\right)(u+v)-f\left(x,|u|^{2}\right) u\right| \leq$ $C\left(1+|u|^{p-2}+|v|^{p-2}\right)|v|$ holds for all $u, v \in \mathbf{C}$. Here, the constant $p$ is as in (f.2).

Throughout this paper, we denote by $\nabla_{\lambda A}$ the distributional covariant derivative $\nabla-i \lambda A$. For any $\lambda>0$, we introduce the Banach space

$$
E_{\lambda}=\left\{u \in L^{2}\left(\mathbf{R}^{N}\right) \mid \nabla_{\lambda A} u \in L^{2}\left(\mathbf{R}^{N}, \mathbf{C}^{N}\right)\right\}
$$

with norm $\|u\|_{\lambda}=\left\|\nabla_{\lambda A} u\right\|_{L^{2}\left(\mathbf{R}^{N}, \mathbf{C}^{N}\right)}=\left(\sum_{j=1}^{N}\left\|\left(\partial_{j}-i \lambda A_{j}\right) u\right\|_{L^{2}\left(\mathbf{R}^{N}\right)}^{2}\right)^{1 / 2}$. We define the functional

$$
J_{\lambda}(u)=\frac{1}{2}\|(\nabla-i \lambda A) u\|_{L^{2}\left(\mathbf{R}^{N}, \mathbf{C}^{N}\right)}^{2}-\int_{\mathbf{R}^{N}} F\left(x,|u|^{2}\right) d x
$$

on $E_{\lambda}$. It is standard that any critical point $u$ of $J_{\lambda}$ is a weak solution to the equation (1.1), i.e., $u$ satisfies $\left(\nabla_{\lambda A} u, \nabla_{\lambda A} v\right)_{L^{2}\left(\mathbf{R}^{N}, \mathbf{C}^{N}\right)}=\int_{\mathbf{R}^{N}} f\left(x,|u|^{2}\right) u \bar{v} d x$ for any $v \in E_{\lambda}$.

The main results of this paper are the following three theorems.

THEOREM 1.1. Assume (A.1)-(A.5) and (f.1)-(f.4). For any integer $m \geq 1$, there exists $\Lambda_{m}>0$ such that the equation (1.1) has at least $m$ weak solutions $\left\{v_{j}^{\lambda}\right\}_{j=1}^{m}$ in $E_{\lambda}$ for any $\lambda \geq \Lambda_{m}$.

Moreover, for any $j=1,2, \ldots, m$, there exist $\lambda$-independent constants $a_{j}, b_{j}$, and $c_{j}$ such that $0<a_{j} \leq\left\|v_{j}^{\lambda}\right\|_{L^{p}\left(\mathbf{R}^{N}\right)} \leq b_{j}<a_{j+1}$ and $\left\|v_{j}^{\lambda}\right\|_{\lambda} \leq c_{j}$ for all $\lambda \geq \Lambda_{m}$. Here, the constant $p$ is as in (f.2).

Define $I_{\Omega}(u)=\frac{1}{2}\|\nabla u\|_{L^{2}(\Omega)}^{2}-\int_{\Omega} F\left(x,|u|^{2}\right) d x$ for $u \in H_{0}^{1}(\Omega)$. Throughout this paper, we regard the space $H_{0}^{1}(\Omega)$ as a closed subspace of $H^{1}\left(\mathbf{R}^{N}\right)$ by zero extension.

THEOREM 1.2. Assume (A.1)-(A.5) and (f.1)-(f.4). Let $\left\{\lambda_{n}\right\}_{n=1}^{\infty}$ be a sequence such that $\lambda_{n} \rightarrow \infty$ as $n \rightarrow \infty$. Assume that, for every $n$, the equation (1.1) with $\lambda=\lambda_{n}$ has a weak solution $u_{n}$ in $E_{\lambda_{n}}$ satisfying: $\sup _{n}\left\|u_{n}\right\|_{\lambda_{n}}<\infty$ and $\inf _{n}\left\|u_{n}\right\|_{L^{p}\left(\mathbf{R}^{N}\right)}>0$. Then there exist a subsequence $\left\{u_{n(k)}\right\}_{k}$ of $\left\{u_{n}\right\}_{n}$ and $v \in H_{0}^{1}(\Omega)$ such that $\left\|u_{n(k)}-v\right\|_{\lambda_{n(k)}} \rightarrow 0$; $u_{n(k)} \rightarrow v$ in $L^{q}\left(\mathbf{R}^{N}\right)$ for every $q \in\left[2,2^{*}\right) ; J_{\lambda_{n(k)}}\left(u_{n(k)}\right) \rightarrow I_{\Omega}(v) ;$ and $v$ is a nontrivial weak solution to

$$
-\Delta v=f\left(x,|v|^{2}\right) v .
$$

Define $\mathcal{N}_{\lambda}=\left\{\left.u \in E_{\lambda} \backslash\{0\}\right|_{E_{\lambda}^{\prime}}\left\langle J_{\lambda}^{\prime}(u), u\right\rangle_{E_{\lambda}}=0\right\}$ and $c_{\lambda}=\inf _{\mathcal{N}_{\lambda}} J_{\lambda}$. Define $\mathcal{N}_{\Omega}=\{u \in$ $\left.\left.H_{0}^{1}(\Omega) \backslash\{0\}\right|_{H_{0}^{1}(\Omega)^{\prime}}\left\langle I_{\Omega}^{\prime}(u), u\right\rangle_{H_{0}^{1}(\Omega)}=0\right\}$ and $c(\Omega)=\inf _{\mathcal{N}_{\Omega}} I_{\Omega}$.

It is well-known that $c(\Omega)$ is positive and attained by some critical point of $I_{\Omega}$.

THEOREM 1.3. Assume (A.1)-(A.5) and (f.1)-(f.6). Then there exists $\Lambda>0$ such that for any $\lambda \geq \Lambda$, the value $c_{\lambda}$ is attained by some critical point $u_{\lambda} \in E_{\lambda}$ of $J_{\lambda}$. Moreover, $\lim _{\lambda \rightarrow \infty} c_{\lambda}=c(\Omega)$. 
REMARK 1.4. (i) We give examples of $A$ which satisfy all the conditions (A.1)(A.5) with $A^{-1}(0)=B^{-1}(0)=B_{1}(0)$. Fix $\chi \in C^{\infty}([0, \infty))$ satisfying: $0 \leq \chi \leq 1$ and $0 \leq \chi^{\prime} \leq 1$ on $[0, \infty) ; \chi(r)=0$ if and only if $0 \leq r \leq 1 ; \chi(r)=1$ if $r \geq 4 ; \chi$ is increasing. Then the examples are given by $A(x)=\chi\left(|x|^{2}\right)\left(-x_{2}, x_{1}\right) / 2$, where $x=\left(x_{1}, x_{2}\right) \in \mathbf{R}^{2}$ for $N=2$, and $A(x)=\chi\left(|x|^{2}\right)\left(-x_{2}, x_{1}, 0\right) / 2+\left(0,0, \chi\left(x_{3}\right)\right)$ for $N=3$. Similar examples can be constructed in higher dimensions.

(ii) The power-type nonlinearity $f(t)=t^{(p-2) / 2}$, where $2<p<2^{*}$, satisfies all the conditions (f.1)-(f.6).

(iii) Let $\left\{v_{j}^{\lambda}\right\}$ be the solutions obtained in Theorem 1.1. Let $\lambda_{n} \rightarrow \infty$ as $n \rightarrow \infty$. Then for each $j$, the sequence $\left\{u_{n}\right\}_{n}=\left\{v_{j}^{\lambda_{n}}\right\}_{n}$ satisfies the assumption as in Theorem 1.2.

(iv) All of the statements of the theorems above remain valid for the equation $-(\nabla-$ $i \lambda A)^{2} u+\mu u=f\left(x,|u|^{2}\right) u$ for any $\mu<\mu_{1}$, where $\mu_{1}$ is the first eigenvalue of the Dirichlét Laplacian on $\Omega$. Proofs are similar.

The organization of this paper is as follows. In Section 2, we formulate the AvronHerbst-Simon type formulae for the quadratic form $\|u\|_{\lambda}^{2}$. In Section 3, we give preliminary results; some basic properties of the functional $J_{\lambda}$, a spectral concentration of $-(\nabla-i \lambda A)^{2}$ as $\lambda \rightarrow \infty$, and a variation of fountain theorem. In Sections 4,5 , and 6 , we give proofs of Theorems 1.1, 1.2, and 1.3, respectively.

\section{Lower estimates of quadratic forms}

We assume (A.1)-(A.5) in the following. Let $E_{\lambda}$ be as in (1.4). Let $H(\lambda)$ be the unique self-adjoint operator associated with the form $\left\|\nabla_{\lambda A} u\right\|_{L^{2}\left(\mathbf{R}^{N}, \mathbf{C}^{N}\right)}^{2}$ on $C_{0}^{\infty}\left(\mathbf{R}^{N}\right)$. Then $H(\lambda)$ is essentially self-adjoint on $C_{0}^{\infty}(\Omega)$, and $E_{\lambda}$ is the form domain of $H(\lambda)$ (e.g., [Le-Si]).

The proof of the next lemma is elementary.

LEMMA 2.1. Let $K$ be an open subset of $\mathbf{R}^{N}$ and let $\zeta \in C_{0}^{1}(K)$. Then we have the following assertions for any $u \in E_{\lambda}$ :

(i) $\nabla_{\lambda_{A}}\left(\left.u\right|_{K}\right)=\left.\left(\nabla_{\lambda_{A}} u\right)\right|_{K}$ in the sense of distribution.

(ii) If $K$ is bounded, then $\zeta u$ belongs to $H_{0}^{1}(K)$ and satisfies $\|\zeta u\|_{H^{1}(K)} \leq C_{\lambda}\left(\|u\|_{\lambda}+\right.$ $\left.\|u\|_{L^{2}\left(\mathbf{R}^{N}\right)}\right)$. Here, $C_{\lambda}$ is independent of $u$.

Define $\beta_{i j}(x)=B_{i j}(x) /|B(x)|$ for $x \in \mathbf{R}^{N} \backslash B^{-1}(0)$ and $1 \leq i, j \leq N$. We note that $\gamma_{B}(x)=\left(\sum_{i<j}\left|\nabla \beta_{i j}(x)\right|^{2}\right)^{1 / 2}$.

Lemma 2.2 ([He-Na], Lemma B.3). Let $\xi=\left(\xi_{i j}\right)_{i, j}$ be a (constant) real antisymmetric matrix with $\sum_{i<j}\left|\xi_{i j}\right|^{2}=1$. We have the inequality

$$
H(\lambda) \geq \sum_{l<m} \lambda \xi_{l m} B_{l m}
$$


in the sense of the sesqui-linear form.

Proposition 2.3. Assume that $F$ is a closed subset of $\mathbf{R}^{N}$ and $B^{-1}(0) \subset F$. Assume that $0<s<\delta$ and $0<s<1 / \sup _{\left(F_{\delta-s}^{+}\right)} \gamma_{B}$. Then

$$
H(\lambda) \geq \lambda|B|\left(1-s\left[\sup _{\left(F_{\delta-s}^{+}\right)^{c}} \gamma_{B}\right]\right) 1_{\left(F_{\delta+s}^{+}\right)^{c}}-c_{0} / s^{2}
$$

where

$$
c_{0}=\inf \left\{\|\nabla \mu\|_{L^{2}\left(\mathbf{R}^{N}, \mathbf{R}^{N}\right)}^{2} /\|\mu\|_{L^{2}\left(\mathbf{R}^{N}\right)}^{2} \mid \mu \in C_{0}^{\infty}\left(\operatorname{int} B_{1}(0), \mathbf{R}\right) \backslash\{0\}\right\},
$$

and $1_{S}$ stands for the characteristic function on $S$.

PRoOF. The proof is essentially the same as that of Proposition B.1 in [He-Na]; however, we give a proof for the sake of completeness.

Take $\mu \in C_{0}^{\infty}\left(\operatorname{int} B_{1}(0), \mathbf{R}\right)$ with $\|\mu\|_{L^{2}}=1$. For any $x_{0} \in \mathbf{R}^{N}$ and $s>0$, we define $\mu_{s, x_{0}}(x)=s^{-N / 2} \mu\left(\left(x-x_{0}\right) / s\right)$. Then $\operatorname{supp}\left(\mu_{s, x_{0}}\right) \subset B_{s}\left(x_{0}\right), \int_{\mathbf{R}^{N}}\left|\mu_{s, x_{0}}(x)\right|^{2} d x_{0}=1$ and $\int_{\mathbf{R}^{N}}\left|\nabla_{x} \mu_{s, x_{0}}(x)\right|^{2} d x_{0}=s^{-2}\|\nabla \mu\|_{L^{2}\left(\mathbf{R}^{N}, \mathbf{R}^{N}\right)}^{2}$ for all $x \in \mathbf{R}^{N}$.

Let $0<s<\delta$ and $x_{0} \in\left(F_{\delta}^{+}\right)^{c}$. By Lemma 2.2 with $\xi_{i j}=\beta_{i j}\left(x_{0}\right)$, we have

$$
\begin{aligned}
H(\lambda) & \geq \sum_{l<m} \lambda \beta_{l m}\left(x_{0}\right) B_{l m}(x) \\
& =\lambda\left(|B(x)|+\sum_{l<m}\left(\beta_{l m}\left(x_{0}\right)-\beta_{l m}(x)\right) B_{l m}(x)\right) \\
& \geq \lambda|B(x)|\left(1-\left[\sum_{l<m}\left|\beta_{l m}(x)-\beta_{l m}\left(x_{0}\right)\right|^{2}\right]^{1 / 2}\right) .
\end{aligned}
$$

By the mean-value theorem, for any $x \in B_{s}\left(x_{0}\right)$,

$$
\begin{aligned}
\left|\beta_{l m}(x)-\beta_{l m}\left(x_{0}\right)\right| & \leq\left|x-x_{0}\right| \int_{0}^{1}\left|\nabla \beta_{l m}\left(t x+(1-t) x_{0}\right)\right| d t \\
& \leq\left|x-x_{0}\right|\left(\int_{0}^{1}\left|\nabla \beta_{l m}\left(t x+(1-t) x_{0}\right)\right|^{2} d t\right)^{1 / 2} .
\end{aligned}
$$

Therefore, it follows from the definition of $\gamma_{B}$ that

$$
\left[\sum_{l<m}\left|\beta_{l m}(x)-\beta_{l m}\left(x_{0}\right)\right|^{2}\right]^{1 / 2} \leq s \sup _{B_{s}\left(x_{0}\right)} \gamma_{B}
$$

for all $x \in B_{S}\left(x_{0}\right)$. 
On the other hand, using the IMS type formula, which can be verified by direct computation, we have

$$
\frac{1}{2}\left(\left(\mu_{s, x_{0}}\right)^{2} H(\lambda)+H(\lambda)\left(\mu_{s, x_{0}}\right)^{2}\right)=\mu_{s, x_{0}} H(\lambda) \mu_{s, x_{0}}-\left|\nabla_{x} \mu_{s, x_{0}}\right|^{2}
$$

as a sesqui-linear form on $C_{0}^{\infty}\left(\mathbf{R}^{N}\right) \times C_{0}^{\infty}\left(\mathbf{R}^{N}\right)$. Integrating this with respect to $x_{0}$ over $\mathbf{R}^{N}$, we have, from (2.1) and (2.2),

$$
\begin{aligned}
H(\lambda) & =\int_{\mathbf{R}^{N}} \mu_{s, x_{0}} H(\lambda) \mu_{s, x_{0}} d x_{0}-s^{-2}\|\nabla \mu\|_{L^{2}\left(\mathbf{R}^{N}, \mathbf{R}^{N}\right)}^{2} \\
& \geq \int_{\left(F_{\delta}^{+}\right)^{c}} \mu_{s, x_{0}} H(\lambda) \mu_{s, x_{0}} d x_{0}-s^{-2}\|\nabla \mu\|_{L^{2}\left(\mathbf{R}^{N}, \mathbf{R}^{N}\right)}^{2} \\
& \geq \lambda|B| \int_{\left(F_{\delta}^{+}\right)^{c}}\left(1-s\left[\sup _{B_{s}\left(x_{0}\right)} \gamma_{B}\right]\right)\left|\mu_{s, x_{0}}\right|^{2} d x_{0}-s^{-2}\|\nabla \mu\|_{L^{2}\left(\mathbf{R}^{N}, \mathbf{R}^{N}\right)}^{2} \\
& \geq \lambda|B|\left(1-s\left[\sup _{\left(F_{\delta-s}^{+}\right)^{c}} \gamma_{B}\right]\right) \int_{\left(F_{\delta}^{+}\right)^{c}}\left|\mu_{s, x_{0}}\right|^{2} d x_{0}-s^{-2}\|\nabla \mu\|_{L^{2}\left(\mathbf{R}^{N}, \mathbf{R}^{N}\right)}^{2} \\
& =\lambda|B|\left(1-s\left[\sup _{\left(F_{\delta-s}^{+}\right)^{c}} \gamma_{B}\right]\right)\left(1-\int_{F_{\delta}^{+}}\left|\mu_{s, x_{0}}\right|^{2} d x_{0}\right)-s^{-2}\|\nabla \mu\|_{L^{2}\left(\mathbf{R}^{N}, \mathbf{R}^{N}\right)}^{2} \\
& \geq \lambda|B|\left(1-s\left[\sup _{\left(F_{\delta-s}^{+}\right)^{c}} \gamma_{B}\right]\right) 1_{\left(F_{\delta+s}^{+}\right)^{c}-s^{-2}\|\nabla \mu\|_{L^{2}\left(\mathbf{R}^{N}, \mathbf{R}^{N}\right)}^{2} .}
\end{aligned}
$$

Here, the last inequality follows from the contraposition of the inclusion $\left(\operatorname{supp}\left(\mu_{s, x_{0}}\right) \subset\right)$ $B_{s}\left(x_{0}\right) \subset F_{\delta+s}^{+}$for $x_{0} \in F_{\delta}^{+}$.

Finally, taking the infimum over $\mu$ on both sides of (2.3), we have the conclusion.

LemMA 2.4. Let $R>0$ satisfy $B^{-1}(0) \subset \operatorname{int}\left(B_{R}(0)\right)$. There exist $\Lambda_{R}>0$ and $C_{R}>0$ such that $\|u\|_{L^{2}\left(B_{R}(0)\right)}^{2} \leq C_{R}\|u\|_{\lambda}^{2}$ and $\lambda\|u\|_{L^{2}\left(B_{R}(0)^{c}\right)}^{2} \leq C_{R}\|u\|_{\lambda}^{2}$ for any $u \in E_{\lambda}$ and any $\lambda \geq \Lambda_{R}$.

In particular, there exist $\Lambda>0$ and $C>0$ such that $\|u\|_{L^{2}\left(\mathbf{R}^{N}\right)} \leq C\|u\|_{\lambda}$ for any $u \in E_{\lambda}$ and any $\lambda \geq \Lambda$.

Proof. Let $r>0$ satisfy that $B^{-1}(0) \subset \operatorname{int}\left(B_{r}(0)\right)$, and let $\varepsilon>0$ small enough. Set $\delta=\varepsilon \operatorname{dist}\left(B^{-1}(0), \partial B_{r}(0)\right)$ and $s=\min \left\{\delta, 1 / \sup _{B_{r-\delta}(0)^{c}} \gamma_{B}\right\} / 3$, which are positive by (A.2) and (A.3). By Proposition 2.3 with $F=B_{r}(0)$, we have

$$
\begin{aligned}
\|u\|_{\lambda}^{2} & \geq \lambda\left(\inf _{\left(F_{\delta+s}^{+}\right)^{c}}|B|\right)\left(1-s \sup _{\left(F_{\delta-s}^{+}\right)^{c}} \gamma_{B}\right)\|u\|_{L^{2}\left(\left(F_{\delta+s}^{+}\right)^{c}\right)}^{2}-\left(c_{0} / s^{2}\right)\|u\|_{L^{2}\left(\mathbf{R}^{N}\right)}^{2} \\
& =: \lambda C\|u\|_{L^{2}\left(\left(F_{\delta+s}^{+}\right)^{c}\right)}^{2}-c\|u\|_{L^{2}\left(\mathbf{R}^{N}\right)}^{2}
\end{aligned}
$$




$$
=(\lambda C-c)\|u\|_{L^{2}\left(\left(F_{\delta+s}^{+}\right)^{c}\right)}^{2}-c\|u\|_{L^{2}\left(F_{\delta+s}^{+}\right)}^{2}
$$

for any $u \in E_{\lambda}$. Hence,

$$
\|u\|_{L^{2}\left(\left(F_{\delta+s}^{+}\right)^{c}\right)}^{2} \leq(\lambda C-c)^{-1}\left(c\|u\|_{L^{2}\left(F_{\delta+s}^{+}\right)}^{2}+\|u\|_{\lambda}^{2}\right) .
$$

Take and fix $\zeta_{r} \in C_{0}^{\infty}\left(\mathbf{R}^{N}\right)$ such that $0 \leq \zeta_{r} \leq 1$ on $\mathbf{R}^{N}, \zeta_{r}=1$ on $B_{r+2 \delta}$, and $\operatorname{supp}\left(\zeta_{r}\right) \subset \operatorname{int}\left[B_{2 r}(0)\right]$. Since $\left|\zeta_{r} u\right| \in H_{0}^{1}\left(\operatorname{int}\left[B_{2 r}(0)\right]\right)$ for any $u \in E_{\lambda}$ by Lemma 2.1 (ii), it follows from the Poincaré and the dia-magnetic inequalities that

$$
\begin{aligned}
\|u\|_{L^{2}\left(F_{\delta+s}^{+}\right)}^{2} & \leq\left\|\zeta_{r} u\right\|_{L^{2}\left(B_{2 r}(0)\right)}^{2} \leq C\left\|\nabla\left|\zeta_{r} u\right|\right\|_{L^{2}\left(B_{2 r}(0), \mathbf{R}^{N}\right)}^{2} \\
& \leq C\left\|\nabla_{\lambda A}\left(\zeta_{r} u\right)\right\|_{L^{2}\left(B_{2 r}(0), \mathbf{C}^{N}\right)}^{2} \leq C^{\prime \prime}\left(\|u\|_{L^{2}\left(B_{2 r}(0) \backslash F_{\delta+s}^{+}\right)}^{2}+\|u\|_{\lambda}^{2}\right) \\
& \leq C^{\prime \prime}\left(\|u\|_{L^{2}\left(\left(F_{\delta+s}^{+}\right)^{c}\right)}^{2}+\|u\|_{\lambda}^{2}\right) \\
& \leq C^{\prime \prime} c(\lambda C-c)^{-1}\|u\|_{L^{2}\left(F_{\delta+s}^{+}\right)}^{2}+C^{\prime \prime}\left(1+(\lambda C-c)^{-1}\right)\|u\|_{\lambda}^{2},
\end{aligned}
$$

where we used (2.4) in the last inequality. Hence,

$$
\left(\lambda C-c-C^{\prime \prime} c\right)\|u\|_{L^{2}\left(F_{\delta+s}^{+}\right)}^{2} \leq C^{\prime \prime}(\lambda C-c+1)\|u\|_{\lambda}^{2} .
$$

Since $r$ and $\varepsilon$ are arbitrary under the restrictions described above and $F_{\delta+s}^{+}=B_{r+\delta+s}(0)$, we deduce the lemma from (2.4) and (2.5).

COROLlaRY 2.5. Let $r>0$ be fixed. Let $\left\{y_{n}\right\}_{n}$ be a sequence in $\mathbf{R}^{N}$ such that $\lambda_{n} \rightarrow$ $\infty$ as $n \rightarrow \infty$. There exist $\Lambda^{\prime}>0, N^{\prime}>0$, and $C^{\prime}>0$ such that

$$
\|u\|_{\lambda}^{2} \geq \lambda C^{\prime}\|u\|_{L^{2}\left(B_{r}\left(y_{n}\right)\right)}^{2}
$$

for any $\lambda \geq \Lambda^{\prime}$, any $u \in E_{\lambda}$, and any $n \geq N^{\prime}$.

COROLlaRY 2.6. Let $\delta>0$ and $F=\left[B^{-1}(0)\right]_{\delta}^{+}$. There exists $C_{\delta}>0$, independent of large $\lambda$, such that $\lambda\|u\|_{L^{2}\left(F^{c}\right)}^{2} \leq C_{\delta}\|u\|_{\lambda}^{2}$ for any $u \in E_{\lambda}$.

Proof. Let $\delta>0$ and $F=B^{-1}(0)$. Fix $R>0$ so large that $\left[B^{-1}(0)\right]_{\delta}^{+} \subset \operatorname{int}\left(B_{R}(0)\right)$. By (A.2), (A.3) and Proposition 2.3, for any small $s>0$, we have

$$
\begin{aligned}
\|u\|_{\lambda}^{2} & \geq \lambda\left(\inf _{\left(F_{\delta+s}^{+}\right)^{c}}|B|\right)\left(1-s \sup _{\left(F_{\delta-s}^{+}\right)^{c}} \gamma_{B}\right)\|u\|_{L^{2}\left(\left(F_{\delta+s}^{+}\right)^{c}\right)}^{2}-\left(c_{0} / s^{2}\right)\|u\|_{L^{2}\left(\mathbf{R}^{N}\right)}^{2} \\
& =(\lambda C-c)\|u\|_{L^{2}\left(\left(F_{\delta+s}^{+}\right)^{c}\right)}^{2}-c\|u\|_{L^{2}\left(F_{\delta+s}^{+}\right)}^{2} \\
& \geq(\lambda C-c)\|u\|_{L^{2}\left(\left(F_{\delta+s}^{+}\right)^{c}\right)}^{2}-c\|u\|_{L^{2}\left(B_{R}(0)\right)}^{2} \\
& \geq(\lambda C-c)\|u\|_{L^{2}\left(\left(F_{\delta+s}^{+}\right)^{c}\right)}^{2}-c C_{R}\|u\|_{\lambda}^{2}
\end{aligned}
$$


for any $u \in E_{\lambda}$, where we used Lemma 2.4 in the last inequality. The arbitrariness of $\delta$ (and small $s$ ) shows the lemma.

LEMMA 2.7. For any $\lambda \geq \Lambda$, where $\Lambda$ is as in Lemma 2.4, we have the following assertions:

(i) The Banach space $E_{\lambda}$ is continuously embedded into $L^{q}\left(\mathbf{R}^{N}\right)$ for any $q \in\left[2,2^{*}\right]$ if $N \geq 3$ and for any $q \geq 2$ if $N=2$.

(ii) For any bounded measurable subset $K$ of $\mathbf{R}^{N}$, the restriction map $E_{\lambda} \rightarrow L^{q}(K)$ is compact for any $q \in\left[2,2^{*}\right)$ if $N \geq 3$ and for any $q \geq 2$ if $N=2$.

ProOF. Let $q$ be as above. It follows from the Sobolev and the dia-magnetic inequalities, and Lemma 2.4 that

$$
\begin{aligned}
\|u\|_{L^{q}\left(\mathbf{R}^{N}\right)} & \leq C_{N, q}\||u|\|_{H^{1}\left(\mathbf{R}^{N}\right)} \leq C_{N, q}\left(\left\|\nabla_{\lambda A} u\right\|_{L^{2}\left(\mathbf{R}^{N}, \mathbf{C}^{N}\right)}^{2}+\|u\|_{L^{2}\left(\mathbf{R}^{N}\right)}^{2}\right)^{1 / 2} \\
& \leq C_{N, q}\left(1+C^{2}\right)^{1 / 2}\|u\|_{\lambda}
\end{aligned}
$$

for any $u \in E_{\lambda}$ and any $\lambda \geq \Lambda$. Here, $C_{N, q}$ and $C$ are independent of $\lambda$. This shows (i).

Next, we show (ii). Let $K$ and $q$ be as in (ii) above. Choose $R>0$ such that $K \subset B_{R}(0)$, and take and fix $\zeta_{K} \in C_{0}^{\infty}\left(\mathbf{R}^{N}\right)$ such that $0 \leq \zeta_{K} \leq 1$ on $\mathbf{R}^{N}, \zeta_{K}=1$ on $K$, and $\operatorname{supp}\left(\zeta_{K}\right) \subset$ int $\left[B_{2 R}(0)\right]$.

The map $T_{1}: E_{\lambda} \ni u \mapsto \zeta_{R} u \in H_{0}^{1}\left(\operatorname{int}\left[B_{2 R}(0)\right]\right)$ is continuous by Lemmata 2.1 and 2.4, the embedding $T_{2}: H_{0}^{1}\left(\operatorname{int}\left[B_{2 R}(0)\right]\right) \rightarrow L^{q}\left(B_{2 R}(0)\right)$ is compact by the Rellich-Kondrashov theorem, and the restriction $T_{3}: L^{q}\left(B_{2 R}(0)\right) \rightarrow L^{q}(K)$ is continuous. Thus, the composition $T_{3} \circ T_{2} \circ T_{1}:\left.E_{\lambda} \ni u \mapsto u\right|_{K} \in L^{q}(K)$ is compact for each $\lambda \geq \Lambda$. This completes the proof.

In the rest of this paper, we always assume the condition $\lambda \geq \Lambda$, where $\Lambda$ be as in Lemma 2.4 , in the place where $E_{\lambda}$ is under consideration.

To apply the standard variational calculus, except for Subsection 3.2, we shall regard $E_{\lambda}$ as a real Hilbert space equipped with the inner product $(u, v)_{\lambda}=\operatorname{Re}\left(\nabla_{\lambda A} u, \nabla_{\lambda A} v\right)_{L^{2}\left(\mathbf{R}^{N}, \mathbf{C}^{N}\right)}$.

It follows from (A.5) that $\nabla_{\lambda A} u=\nabla u$ and $(u, v)_{\lambda}=\operatorname{Re}(\nabla u, \nabla v)_{L^{2}\left(\Omega, \mathbf{C}^{N}\right)}$ hold for any $u, v \in H_{0}^{1}(\Omega)$. Therefore, we may regard the space $H_{0}^{1}(\Omega)$ as a closed subspace of the Hilbert space $E_{\lambda}$ by zero extension.

\section{Preliminaries}

3.1. Variational structure of $J_{\lambda}$. In this subsection, we recall some (well-known) variational properties of $J_{\lambda}$. Assume (A.1)-(A.5) and (f.1)-(f.4). Let $p \in\left(2,2^{*}\right)$ and $\theta>2$ be as in (f.2) and (f.3).

LEMmA 3.1. (i) There exist $C>0$ and $C^{\prime}>0$ such that $\inf _{x \in \mathbf{R}^{N}} F\left(x, t^{2}\right) \geq C t^{\theta}-$ $C^{\prime} t^{2}$ for any $t \geq 0$. 
(ii) For any $\varepsilon>0$, there exists $C_{\varepsilon}>0$ such that

$$
0<\theta F\left(x, t^{2}\right) \leq f\left(x, t^{2}\right) t^{2} \leq \varepsilon t^{2}+C_{\varepsilon} t^{p}
$$

for any $t>0$ and any $x \in \mathbf{R}^{N}$.

(iii) For any $\varepsilon>0$, there exists $C_{\varepsilon}>0$ such that

$$
\begin{aligned}
& \int_{\mathbf{R}^{N}} f\left(x,|u|^{2}\right)\left|v\|w \mid d x \leq \varepsilon\| v\left\|_{L^{2}\left(\mathbf{R}^{N}\right)}\right\| w \|_{L^{2}\left(\mathbf{R}^{N}\right)}\right. \\
& +C_{\varepsilon}\|u\|_{L^{p}\left(\mathbf{R}^{N}\right)}^{p-2}\|v\|_{L^{p}\left(\mathbf{R}^{N}\right)}\|w\|_{L^{p}\left(\mathbf{R}^{N}\right)}, \\
& \int_{\mathbf{R}^{N}} f\left(x,|u|^{2}\right)|u|^{2} d x+\int_{\mathbf{R}^{N}} F\left(x,|u|^{2}\right) d x \leq \varepsilon\|u\|_{L^{2}\left(\mathbf{R}^{N}\right)}^{2}+C_{\varepsilon}\|u\|_{L^{p}\left(\mathbf{R}^{N}\right)}^{p}, \\
& \int_{\mathbf{R}^{N}} f\left(x,|u|^{2}\right)|u|^{2} d x+\int_{\mathbf{R}^{N}} F\left(x,|u|^{2}\right) d x \leq \varepsilon\|u\|_{\lambda}^{2}+C_{\varepsilon}\|u\|_{\lambda}^{p}
\end{aligned}
$$

for any $u, v, w \in E_{\lambda}$. Here, $C_{\varepsilon}$ is independent of large $\lambda$.

(iv) If $u$ converges to $v$ in $L^{2}\left(\mathbf{R}^{N}\right)$ and in $L^{p}\left(\mathbf{R}^{N}\right)$, then $\int_{\mathbf{R}^{N}} F\left(x,|u|^{2}\right) d x$ tends to $\int_{\mathbf{R}^{N}} F\left(x,|v|^{2}\right) d x$.

PRoOF. First, we show (ii). By (f.2), for any $\varepsilon>0$ there exists $t_{\varepsilon}>0$ such that $f\left(x, t^{2}\right) t^{2} \leq \varepsilon t^{2}$ for all $t \in\left(0, t_{\varepsilon}\right]$ and all $x \in \mathbf{R}^{N}$, and $f\left(x, t^{2}\right) t^{2} \leq C\left(t^{2}+t^{p}\right) \leq$ $C\left(t_{\varepsilon}^{-(p-2)}+1\right) t^{p}=: C_{\varepsilon} t^{p}$ for all $t \geq t_{\varepsilon}$ and all $x \in \mathbf{R}^{N}$. The rest of the assertion follows from (f.3).

Second, we show (i). Let $t_{0}>0$ be as in (f.4). For $t \geq t_{0}$, we have $F(x, t) \geq C t^{\theta / 2}$ by integrating the inequality in (f.3). For $t<t_{0}$, the assertion follows from (ii).

Third, the assertion (iii) follows from (ii), the Hölder inequality, and (2.6).

Finally, we show (iv). For any $\varepsilon>0$ there exists $C_{\varepsilon}>0$ such that $\left.|| u\right|^{2}-|v|^{2} \mid \leq$ $|u-v|(|u|+|v|) \leq C_{\varepsilon}{ }^{-1}|u-v|^{2}+\varepsilon\left(|u|^{2}+|v|^{2}\right)$. Then it follows from the mean-value theorem and (ii) with $\varepsilon=1$ that $2\left|F\left(x,|u|^{2}\right)-F\left(x,|v|^{2}\right)\right| \leq \mid\left(|u|^{2}-|v|^{2}\right) \int_{0}^{1} f\left(t|u|^{2}+(1-\right.$ $\left.t)|v|^{2}\right) d t \mid \leq C_{p}\left(C_{\varepsilon}|u-v|^{2}+\varepsilon\left(|u|^{2}+|v|^{2}\right)\right)\left(1+|u|^{p-2}+|v|^{p-2}\right)$ for any $\varepsilon>0$. Then the assertion follows from the Hölder inequality $\int|u|^{2}|v|^{p-2} \leq\|u\|_{L^{p}}^{2}\|v\|_{L^{p}}^{p-2}$.

The next lemma is well-known (e.g., the proof of Theorem 1.22 in [Zo-Sc]).

LEMMA 3.2. (i) If $u_{n} \rightarrow v$ in $E_{\lambda}$ as $n \rightarrow \infty$, then

$$
\lim _{n \rightarrow \infty} \int_{\mathbf{R}^{N}} f\left(x,\left|u_{n}\right|^{2}\right) u_{n} \bar{w} d x=\int_{\mathbf{R}^{N}} f\left(x,|v|^{2}\right) v \bar{w} d x
$$

for each $w \in E_{\lambda}$.

(ii) If $u_{n} \rightarrow$ vin $E_{\lambda}$ as $n \rightarrow \infty$, then

$$
\lim _{n \rightarrow \infty} \sup _{w \in E_{\lambda} \backslash\{0\}}\left|\int_{\mathbf{R}^{N}} f\left(x,\left|u_{n}\right|^{2}\right) u_{n} \bar{w} d x-\int_{\mathbf{R}^{N}} f\left(x,|v|^{2}\right) v \bar{w} d x\right| /\|w\|_{\lambda}=0 .
$$


The next lemma follows from a simple calculation.

LEMMA 3.3. Let $p>2, a>0$, and $b>0$. The function $f(x)=a x^{2} / 2-b x^{p} / p$ on $[0, \infty)$ takes the maximum $f\left((a / b)^{1 /(p-2)}\right)=\frac{p-2}{2 p}\left(a^{p} / b^{2}\right)^{1 /(p-2)}$. Moreover, $f(x)>0$ if $0<x<(a / b)^{1 /(p-2)}$.

The next lemma follows from the Hölder inequality.

LeMmA 3.4. Let $1<s<q<r<\infty$. Let $K$ be a measurable subset of $\mathbf{R}^{N}$. We have $\|u\|_{L^{q}(K)} \leq\|u\|_{L^{s}(K)}^{a}\|u\|_{L^{r}(K)}^{1-a}$ for any $u \in L^{s}(K) \cap L^{r}(K)$, where $a=s(r-q) /[q(r-s)] \in$ $(0,1)$.

Let $J_{\lambda}$ be the functional on the real Banach space $E_{\lambda}$ defined by (1.5). It follows from Lemma 3.2 that $J_{\lambda} \in C^{1}\left(E_{\lambda}, \mathbf{R}\right)$ and the Fréchet derivative is given by the dual coupling

$$
E_{\lambda}^{\prime}\left\langle J_{\lambda}^{\prime}(u), v\right\rangle_{E_{\lambda}}=\operatorname{Re}\left((u, v)_{\lambda}-\int_{\mathbf{R}^{N}} f\left(x,|u|^{2}\right) u \bar{v} d x\right)
$$

for any $u, v \in E_{\lambda}$.

LEMMA 3.5. There exist $r>0$ and $b>0$, both of which are independent of $\lambda$, such that $J_{\lambda}(u) \geq b$ if $\|u\|_{\lambda}=r$.

Proof. By Lemma 3.1 (iii) with $\varepsilon=1 / 2$, we have

$$
J_{\lambda}(u)=\frac{1}{2}\|u\|_{\lambda}^{2}-\int_{\mathbf{R}^{N}} F\left(x,|u|^{2}\right) d x \geq \frac{1}{4}\|u\|_{\lambda}^{2}-C_{1 / 2}\|u\|_{\lambda}^{p}
$$

for any $u \in E_{\lambda}$. By Lemma 3.3, the rightmost of (3.1) takes the maximum $\frac{p-2}{2 p}\left[(1 / 2)^{p} /\left(p C_{1 / 2}\right)^{2}\right]^{1 /(p-2)}=: b$ if $\|u\|_{\lambda}=\left[1 /\left(2 p C_{1 / 2}\right)\right]^{1 /(p-2)}=: r$. This proves the lemma.

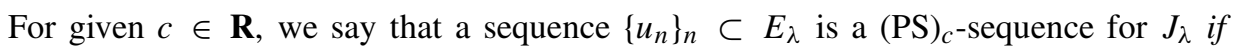
$J_{\lambda}\left(u_{n}\right) \rightarrow c$ and $\left\|J_{\lambda}^{\prime}\left(u_{n}\right)\right\|_{E_{\lambda}^{\prime}} \rightarrow 0$ as $n \rightarrow \infty$. A functional is said to satisfy the (PS) $c^{-}$ condition if any $(\mathrm{PS})_{c}$-sequence has a convergent subsequence.

LEMMA 3.6. For any $(P S)_{c}$-sequence $\left\{u_{n}\right\}_{n} \subset E_{\lambda}$ for $J_{\lambda}$, we have:

(i) $\limsup _{n \rightarrow \infty}\left\|u_{n}\right\|_{\lambda}^{2} \leq[2 \theta /(\theta-2)] c$. In particular, $\left\{u_{n}\right\}_{n}$ is bounded in $E_{\lambda}$ and $c \geq 0$. Here, $\theta$ is as in (f.3).

(ii) For any $q \in\left[2,2^{*}\right]$ for $N \geq 3$ and for any $q \geq 2$ for $N=2$, there exists $C_{q}>0$ such that $\lim \sup _{n \rightarrow \infty}\left\|u_{n}\right\|_{L^{q}\left(\mathbf{R}^{N}\right)}^{2} \leq C_{q}^{2}[2 \theta /(\theta-2)] c$.

(iii) There exists $c_{1}>0$ such that if $c<c_{1}$ then $c=0$.

(iv) There exists $\alpha>0$ such that $\liminf _{n \rightarrow \infty}\left\|u_{n}\right\|_{L^{p}\left(\mathbf{R}^{N}\right)}^{p} \geq \alpha c$.

Here, all the constants $C_{q}, c_{1}$, and $\alpha$ above are independent of $\lambda, c$, and of the choice of the $(\mathrm{PS})_{c}$-sequence. 
PROOF. We give a proof of these standard results for the sake of completeness. Let $\left\{u_{n}\right\}_{n}$ be a (PS) ${ }_{c}$-sequence for $J_{\lambda}$. Then it follows from (f.3) that

$$
\begin{aligned}
& c+o(1)+o(1)\left\|u_{n}\right\|_{\lambda}=J_{\lambda}\left(u_{n}\right)-\frac{1}{\theta} E_{\lambda}^{\prime}\left\langle J_{\lambda}^{\prime}\left(u_{n}\right), u_{n}\right\rangle_{E_{\lambda}} \\
= & (1 / 2-1 / \theta)\left\|u_{n}\right\|_{\lambda}^{2}+\int_{\mathbf{R}^{N}}\left(\frac{1}{\theta} f\left(x,\left|u_{n}\right|^{2}\right)\left|u_{n}\right|^{2}-F\left(x,\left|u_{n}\right|^{2}\right)\right) d x \\
\geq & {[(\theta-2) /(2 \theta)]\left\|u_{n}\right\|_{\lambda}^{2} }
\end{aligned}
$$

as $n \rightarrow \infty$, which implies (i) because $o(1)\left\|u_{n}\right\|_{\lambda} \leq \varepsilon\left\|u_{n}\right\|_{\lambda}^{2}+o(1)$ for any $\varepsilon>0$. Then, applying (2.6) to (i) above, we have (ii). Note that the constant $C_{q}$ from (2.6) depends only on $N$ and $q$.

It follows from Lemma 3.1 (iii) with $\varepsilon=1 / 2$ that

$$
\begin{aligned}
o(1)\left\|u_{n}\right\|_{\lambda} & ={ }_{E_{\lambda}^{\prime}}\left\langle J_{\lambda}^{\prime}\left(u_{n}\right), u_{n}\right\rangle_{E_{\lambda}} \geq\left\|u_{n}\right\|_{\lambda}^{2}-\left(\frac{1}{2}\left\|u_{n}\right\|_{\lambda}^{2}+C_{1 / 2}\left\|u_{n}\right\|_{\lambda}^{p}\right) \\
& =\frac{1}{4}\left\|u_{n}\right\|_{\lambda}^{2}+\left(\frac{1}{4}\left\|u_{n}\right\|_{\lambda}^{2}-C_{1 / 2}\left\|u_{n}\right\|_{\lambda}^{p}\right)
\end{aligned}
$$

as $n \rightarrow \infty$. Thus, by Lemma 3.3 with $a=1 / 2$ and $b=p C_{1 / 2}$, we have

$$
\left\|u_{n}\right\|_{\lambda}^{2} \leq o(1)\left\|u_{n}\right\|_{\lambda}
$$

if $\left\|u_{n}\right\|_{\lambda} \leq\left(2 p C_{1 / 2}\right)^{-1 /(p-2)}$.

We now assume that $c<[(\theta-2) /(2 \theta)]\left(2 p C_{1 / 2}\right)^{-2 /(p-2)}=: c_{1}$. Then it follows from (i) that $\lim \sup _{n \rightarrow \infty}\left\|u_{n}\right\|_{\lambda} \leq([2 \theta /(\theta-2)] c)^{1 / 2}<\left(2 p C_{1 / 2}\right)^{-1 /(p-2)}$, and hence $\lim _{n \rightarrow \infty}\left\|u_{n}\right\|_{\lambda}=0$ by (3.3). Then $J_{\lambda}\left(u_{n}\right) \rightarrow J_{\lambda}(0)=0=c$. This shows (iii).

Finally, we show (iv). By Lemma 3.1, (i), (ii), and (2.6), for any $\varepsilon>0$ there exists $C_{\varepsilon}>0$ such that, as $n \rightarrow \infty$,

$$
\begin{aligned}
c+o(1)+o(1)\left\|u_{n}\right\|_{\lambda} & =J_{\lambda}\left(u_{n}\right)-\frac{1}{2} E_{\lambda}^{\prime}\left\langle J_{\lambda}^{\prime}\left(u_{n}\right), u_{n}\right\rangle_{E_{\lambda}} \\
& =\int_{\mathbf{R}^{N}}\left(\frac{1}{2} f\left(x,\left|u_{n}\right|^{2}\right)\left|u_{n}\right|^{2}-F\left(x,\left|u_{n}\right|^{2}\right)\right) d x \\
& \leq \varepsilon\left\|u_{n}\right\|_{L^{2}\left(\mathbf{R}^{N}\right)}^{2}+C_{\varepsilon}\left\|u_{n}\right\|_{L^{p}\left(\mathbf{R}^{N}\right)}^{p} \\
& \leq \varepsilon C_{2}^{2}\left\|u_{n}\right\|_{\lambda}^{2}+C_{\varepsilon}\left\|u_{n}\right\|_{L^{p}\left(\mathbf{R}^{N}\right)}^{p} .
\end{aligned}
$$

Using (i), we deduce from (3.4) that

$$
\liminf _{n \rightarrow \infty}\left\|u_{n}\right\|_{L^{p}\left(\mathbf{R}^{N}\right)}^{p} \geq C_{\varepsilon}^{-1}\left(1-C_{2}^{2}[2 \theta /(\theta-2)] \varepsilon\right) c .
$$

Choose $\varepsilon>0$ so that $C_{2}^{2}[2 \theta /(\theta-2)] \varepsilon=1 / 2$ and set $\alpha=C_{\varepsilon}^{-1} / 2$. This completes the proof. 


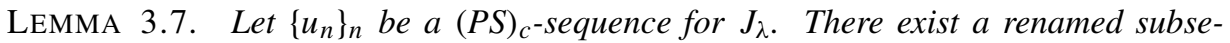
quence $\left\{u_{n}\right\}_{n}$ and $v \in E_{\lambda}$ satisfying: (i) $u_{n} \rightarrow v$ in $E_{\lambda}$; (ii) $u_{n} \rightarrow v$ in $L_{\mathrm{loc}}^{q}\left(\mathbf{R}^{N}\right)$ for any $q \in\left[2,2^{*}\right)$; (iii) $u_{n}(x) \rightarrow v(x)$ for a.e. $x \in \mathbf{R}^{N}$; (iv) $J_{\lambda}^{\prime}(v)=0$; (v) For any $R>0$,

$$
\alpha c-\limsup _{n \rightarrow \infty}\left\|u_{n}\right\|_{L^{p}\left(B_{R}(0)^{c}\right)}^{p} \leq\|v\|_{L^{p}\left(\mathbf{R}^{N}\right)}^{p} \leq\left\{C_{p}^{2}[2 \theta /(\theta-2)] c\right\}^{p / 2} .
$$

Here, the constants $C_{p}$ and $\alpha$ are as in Lemma 3.6 (ii) and (iv).

Proof. By a standard argument, we have (i)-(iii) since $\left\{u_{n}\right\}_{n}$ is bounded in $E_{\lambda}$. The assertion (iv) follows from (i) and Lemma 3.2.

We show (v). Let $\left\{u_{n}\right\}_{n}$ be a renamed subsequence satisfying (i)-(iv). It follows from (i), Fatou's lemma, and Lemma 3.6 (i) that

$$
\|v\|_{L^{p}\left(\mathbf{R}^{N}\right)}^{2} \leq \liminf _{n \rightarrow \infty}\left\|u_{n}\right\|_{L^{p}\left(\mathbf{R}^{N}\right)}^{2} \leq C_{p}^{2}[2 \theta /(\theta-2)] c .
$$

On the other hand, it follow from (ii) and Lemma 3.6 (iv) that, for any $R>0$,

$$
\begin{aligned}
\|v\|_{L^{p}\left(\mathbf{R}^{N}\right)}^{p} & \geq\|v\|_{L^{p}\left(B_{R}(0)\right)}^{p}=\lim _{n \rightarrow \infty}\left\|u_{n}\right\|_{L^{p}\left(B_{R}(0)\right)}^{p} \\
& \geq \liminf _{n \rightarrow \infty}\left\|u_{n}\right\|_{L^{p}\left(\mathbf{R}^{N}\right)}^{p}-\limsup _{n \rightarrow \infty}\left\|u_{n}\right\|_{L^{p}\left(B_{R}(0)^{c}\right)}^{p} \\
& \geq \alpha c-\limsup _{n \rightarrow \infty}\left\|u_{n}\right\|_{L^{p}\left(B_{R}(0)^{c}\right)}^{p}
\end{aligned}
$$

These complete the proof.

3.2. Convergence of spectrum. In this subsection, we give a spectral property which follows from the norm resolvent convergence of the self-adjoint operator $H(\lambda)$ as $\lambda \rightarrow \infty$.

Let $\left\{\mu_{j}\right\}_{j=1}^{\infty}$ be the strictly increasing sequence of all distinct eigenvalues of the Dirichlet Laplacian $-\Delta_{\Omega}$ acting on the complex Hilbert space $L^{2}(\Omega)$ with $\operatorname{Dom}\left(-\Delta_{\Omega}\right)=H^{2}(\Omega) \cap$ $H_{0}^{1}(\Omega)$. We denote by $\operatorname{mult}\left(\mu_{j}\right)$ the multiplicity of the eigenvalue $\mu_{j}$.

LEMMA 3.8. For any integer $k \geq 1$ and any $\varepsilon>0$, there exists $\Lambda_{k, \varepsilon}>0$ such that, for any $\lambda \geq \Lambda_{k, \varepsilon}$,

(i) $H(\lambda)$ has at least $\sum_{j=1}^{k} \operatorname{mult}\left(\mu_{j}\right)$ eigenvalues (counted multiplicities):

$$
\mathcal{E}_{k}:=\bigcup_{j=1}^{k} \bigcup_{l=1}^{\operatorname{mult}\left(\mu_{j}\right)}\left\{\mu_{j, l}^{\lambda}\right\} .
$$

(ii) $\sum_{j=1}^{k} \sum_{l=1}^{\operatorname{mult}\left(\mu_{j}\right)}\left|\mu_{j, l}^{\lambda}-\mu_{j}\right|<\varepsilon$.

(iii) $\operatorname{Spec}(H(\lambda)) \cap\left(-\infty, \mu_{k}+\varepsilon\right]=\mathcal{E}_{k}$.

Proof. This is a consequence of the results obtained by [He-Na] (See also [He-He]).

In fact, since $\bar{\Omega}$ is compact by (A.2), it follows from Theorem 2.2 in [He-Na] that $H(\lambda)$ converges to a self-adjoint operator $K(\lambda)$ as $\lambda \rightarrow \infty$ in the norm resolvent sense. On the 
other hand, since $A=0$ on $\Omega$ by (A.5), it follows from Theorem 3.3 in [He-Na] that $K(\lambda)$ is unitarily equivalent to $-\Delta_{\Omega}$, therefore $\operatorname{Spec}(K(\lambda))=\operatorname{Spec}\left(-\Delta_{\Omega}\right)$. Once we have the norm resolvent convergence, the spectral concentration follows from the standard argument as in the proof of Theorems VIII.23 and VIII.24 in [Re-Si] (or as in Appendix A in [He-Na]).

3.3. Fountain theorem. In this subsection, we formulate a variant of Theorem 3.5 of Willem's monograph [Wi]. The theorem will be used to produce $(\mathrm{PS})_{c}$-sequences for $J_{\lambda}$ for certain $c$ 's.

Let $X$ be a real Banach space. Assume that $X$ has a direct product decomposition $X=$ $\overline{\oplus_{j \geq 1} X_{j}}$, where each $X_{j}$ is a finite-dimensional subspace. For any $k \geq 1$, set $Y_{k}=\oplus_{1 \leq j \leq k} X_{j}$ and $Z_{k}=\overline{\oplus_{j \geq k} X_{j}}$. Clearly, $X=Y_{k}+Z_{k}$ and $Y_{k} \cap Z_{k}=X_{k}$.

Let $G$ be a compact group which acts linearly and isometrically on $X$. Assume that every $X_{j}$ are $G$-invariant. Assume further that for any bounded, $G$-invariant, open neighbourhood $U$ of the origin of $Y_{k+1}$, every continuous $G$-equivariant map $f$ from $\partial U$ to $Y_{k}$ has a zero for every $k \geq 1$.

Then we have the following version of a fountain theorem:

Theorem 3.9. Let $k \geq 1$. Assume that $\varphi \in C^{1}(X, \mathbf{R})$ and $\varphi$ is $G$-invariant. Let $0<r_{k}<\rho_{k}$ and set

$$
c_{k}=\inf _{\gamma \in \Gamma_{k}} \max _{u \in B_{\rho_{k}}(0) \cap Y_{k}} \varphi(\gamma(u)),
$$

where $\Gamma_{k}=\left\{\gamma \in C\left(B_{\rho_{k}}(0) \cap Y_{k}, X\right) \mid \gamma\right.$ is G-equivariant, $\left.\left.\gamma\right|_{\partial\left(B_{\rho_{k}}(0) \cap Y_{k}\right)}=\mathrm{id}\right\}$. Assume that

$$
b_{k}:=\inf _{\partial B_{r_{k}}(0) \cap Z_{k}} \varphi>a_{k}:=\max _{\partial B_{\rho_{k}}(0) \cap Y_{k}} \varphi .
$$

Then we have the following assertions:

(i) $c_{k} \geq b_{k}$, and

(ii) For any $\varepsilon \in\left(0,\left(c_{k}-a_{k}\right) / 2\right)$ and any $\delta>0$, if $\gamma \in \Gamma_{k}$ satisfies $\sup _{u \in B_{\rho_{k}}(0) \cap Y_{k}} \varphi(\gamma(u)) \leq c_{k}+\varepsilon$, then there exists $u \in X$ such that $\left|\varphi(u)-c_{k}\right| \leq 2 \varepsilon$; $\operatorname{dist}\left(u, \gamma\left(B_{\rho_{k}}(0) \cap Y_{k}\right)\right) \leq 2 \delta$; and $\left\|\varphi^{\prime}(u)\right\|_{X^{\prime}} \leq 8 \varepsilon / \delta$.

In Theorem 3.5 of [Wi], it is assumed that $\operatorname{dim} X_{j}$ is independent of $j$. However, its proof applies to Theorem 3.9 above if we use the following intersection lemma instead of Lemma 3.4 in [Wi].

Lemma 3.10. Let $0<r<\rho$. Assume that $\gamma \in C\left(B_{\rho}(0) \cap Y_{k}, X\right)$ is G-equivariant and $\left.\gamma\right|_{\partial B_{\rho}(0) \cap Y_{k}}=i d$. Then $\gamma(0)=0$ and $\gamma\left(B_{\rho}(0) \cap Y_{k}\right) \cap \partial B_{r}(0) \cap Z_{k} \neq \emptyset$.

Proof. Let $\gamma$ be as above. The equivariance of $\gamma$ implies that $\gamma(0) \in Y_{k}^{G}:=\{u \in$ $Y_{k} \mid g(u)=u$ for all $\left.g \in G\right\}$. Note that $g(0)=0$ for all $g \in G$ because $G$ acts linearly. Define a constant map $f: Y_{k+1} \rightarrow Y_{k}^{G}\left(\subset Y_{k}\right)$ by $f(u)=\gamma(0)$, and $\operatorname{set} U=\operatorname{int}\left[B_{1}(0)\right] \cap Y_{k+1}$. By the assumption on the action of $G, \partial U \cap f^{-1}(0) \neq \emptyset$. Hence, $\gamma(0)=0$. 
Set $U=\left\{u \in \operatorname{int}\left[B_{\rho}(0)\right] \cap Y_{k} \mid\|\gamma(u)\|_{X}<r\right\}$, which is a bounded, $G$-invariant, open neighbourhood of the origin of $Y_{k}$. Let $P_{k-1}: X \rightarrow Y_{k-1}$ be the continuous projection and set $f=P_{k-1} \circ \gamma: \bar{U} \rightarrow Y_{k-1}$, which is continuous $G$-equivariant. By the assumption again, there exists $u \in \partial U \cap f^{-1}(0)$. Since $u \in \partial U$, either (i) $\|u\|_{X}=\rho$; or (ii) $\|\gamma(u)\|_{X}=r$. If (i) occurs, then $\gamma(u)=u$ and then $\rho=\|u\|_{X}=\|\gamma(u)\|_{X} \leq r$, which is a contradiction. Thus, $f(u)=0,\|u\|_{X}<\rho$ and $\|\gamma(u)\|_{X}=r$, and then the element $\gamma(u)$ assures the nonemptiness.

In the next section we shall use the antipodal action of $G=\{ \pm 1\}$ on $\mathbf{R}^{n}$ to apply the fountain theorem. In this case, the assumption on the action of $G$ is fulfilled because of the Borsuk-Ulam theorem:

LEMMA 3.11. Let $N>n \geq 0$. Let $U$ be a bounded, symmetric (i.e., invariant under the antipodal action of the group $G=\{ \pm 1\})$, open neighbourhood of the origin of $\mathbf{R}^{N}$. Every continuous odd map $f$ from $\partial U$ to $\mathbf{R}^{n}$ has a zero.

Proof. We may assume that $N>n+1 \geq 2$ because the lemma is trivial if $n=0$ and follows from the standard Borsuk-Ulam theorem (e.g., Theorem D.17 in [Wi]) if $N=n+1$. Define the inclusion $\iota: \mathbf{R}^{n} \rightarrow \mathbf{R}^{N-1}=\mathbf{R}^{n} \times \mathbf{R}^{N-n-1}$ by $\iota(x)=(x ; 0)$. Then the composition $\iota \circ f: \partial U \rightarrow \mathbf{R}^{N-1}$ is continuous and odd. By the Borsuk-Ulam theorem, there exists $x \in \partial U \cap(\iota \circ f)^{-1}(0)$. This implies that $x \in \partial U \cap f^{-1}(0)$.

\section{Proof of Theorem 1.1}

In this section, we show Theorem 1.1. The proof is an adaptation of that of Theorem 1.1 in $[\mathrm{Ba}-\mathrm{Pa}-\mathrm{Wa}]$.

Let $E_{\lambda}$ be the (real) Hilbert space introduced at the end of Section 2, and let $-\Delta_{\Omega},\left\{\mu_{j}\right\}_{j}$, $H(\lambda)$, and $\left\{\mu_{j, l}^{\lambda}\right\}_{j, l}$ be as in Subsection 3.2.

Fix $k \geq 1$ arbitrarily. Set

$$
Y_{k}=\bigoplus_{j=1}^{k} \operatorname{Ker}\left(-\Delta_{\Omega}-\mu_{j}\right)=: \oplus_{j=1}^{k} X_{j}
$$

and

$$
Y_{k}^{\lambda}=\bigoplus_{j=1}^{k} \bigoplus_{l=1}^{\operatorname{mult}\left(\mu_{j}\right)} \operatorname{Ker}\left(H(\lambda)-\mu_{j, l}^{\lambda}\right)
$$

Set $\left(Y_{k}^{\lambda}\right)^{\perp}=\left\{u \in E_{\lambda} \mid(u, v)_{\lambda}=0\right.$ for any $\left.v \in Y_{k}^{\lambda}\right\}$. For notational convenience, we set $Y_{0}=Y_{0}^{\lambda}=\{0\}$ and then $\left(Y_{0}^{\lambda}\right)^{\perp}=E_{\lambda}$. Set $Z_{k}^{\lambda}=E_{\lambda} \ominus Y_{k-1}$.

In what follows, we assume that $\lambda \geq \Lambda_{k+1, \varepsilon}$, where $\Lambda_{k+1, \varepsilon}$ is as in Lemma 3.8 and we set $\varepsilon=\min \left\{\mu_{j+1}-\mu_{j}\right\}_{j=1}^{k} / 100$. 
LEMMA 4.1. There exist $r$ and $R_{k}$, both of which are independent of $\lambda$, such that $0<r<R_{k}$ and

(i)

$$
b_{k}^{\lambda}:=\inf _{\partial B_{r}(0) \cap Z_{k}^{\lambda}} J_{\lambda}>0>-1>a_{k}^{\lambda}:=\max _{\partial B_{R_{k}}(0) \cap Y_{k}} J_{\lambda} .
$$

(ii) $J_{\lambda}(u) \leq 0$ for any $u \in B_{R_{k}}(0)^{c} \cap Y_{k}$.

PROOF. First, the estimate of $b_{k}^{\lambda}$ from below follows from Lemma 3.5 with the common $r$.

Next, we estimate $a_{k}^{\lambda}$ from above. For any $u \in Y_{k}$, we have

$$
\begin{aligned}
J_{\lambda}(u) & =\frac{1}{2}\|u\|_{\lambda}^{2}-\int_{\Omega} F\left(x,|u|^{2}\right) d x \leq \frac{1}{2}\|u\|_{\lambda}^{2}-C\|u\|_{L^{\theta}(\Omega)}^{\theta}+C^{\prime}\|u\|_{L^{2}(\Omega)}^{2} \\
& \leq\left(\frac{1}{2}+C^{\prime \prime}\right)\|u\|_{\lambda}^{2}-C_{k}\|\nabla u\|_{L^{2}\left(\Omega, \mathbf{C}^{N}\right)}^{\theta} \\
& =\left(\frac{1}{2}+C^{\prime \prime}\right)\|u\|_{\lambda}^{2}-C_{k}\|u\|_{\lambda}^{\theta}
\end{aligned}
$$

where we used Lemma 3.1 (i) in the first inequality and used Lemma 2.4 and the equivalence of norms on the $\lambda$-independent finite-dimensional space $Y_{k}$ in the second inequality. Here, $C^{\prime \prime}$ and $C_{k}$ are independent of $\lambda$. Since $\theta>2$, we can take $R_{k}>0$ so large that the rightmost of (4.1) is less than -1 if $\|u\|_{\lambda} \geq R_{k}$. This completes the proof.

Let $R_{k}$ be the constant obtained in Lemma 4.1 above. Set

$$
c_{k}^{\lambda}=\inf _{\gamma \in \Gamma_{k}^{\lambda}} \max _{u \in B_{R_{k}}(0) \cap Y_{k}} J_{\lambda}(\gamma(u)),
$$

where $\Gamma_{k}^{\lambda}=\left\{\gamma \in C\left(B_{R_{k}}(0) \cap Y_{k}, E_{\lambda}\right) \mid \gamma\right.$ is odd, $\left.\left.\gamma\right|_{\partial\left(B_{R_{k}}(0) \cap Y_{k}\right)}=\mathrm{id}\right\}$.

LEMMA 4.2. Let $c_{k}^{\lambda}$ be as in (4.2). There exists a $(P S)_{c_{k}^{\lambda}}$-sequence for $J_{\lambda}$ for every large $\lambda$.

PROOF. We take and fix an orthonormal basis $\left\{\tilde{f}_{m}\right\}_{m=k+1}^{\infty}$ of the subspace $Z_{k+1}^{\lambda}=$ $E_{\lambda} \ominus Y_{k}$ of the Hilbert space $E_{\lambda}$.

We now apply Theorem 3.9 with $X=E_{\lambda} ; G=\{ \pm 1\}$ with the antipodal action; $\varphi=J_{\lambda}$; $X_{j}$ is the space defined at the beginning of this section if $1 \leq j \leq k$, and set $X_{j}=\operatorname{Span}_{\mathbf{C}}\left\{\tilde{f}_{j}\right\}$ if $j \geq k+1 ; Y_{k}$ is the space defined above; $Z_{k}=Z_{k}^{\lambda} ; r_{k}=r ; \rho_{k}=R_{k} ; c_{k}=c_{k}^{\lambda} ; a_{k}=a_{k}^{\lambda}$; $b_{k}=b_{k}^{\lambda} ;$ and $\Gamma_{k}=\Gamma_{k}^{\lambda}$.

The assumption $b_{k}>a_{k}$ in Theorem 3.9 follows from Lemma 4.1. Hence, $c_{k}^{\lambda} \geq b_{k}^{\lambda}$, and for any $\varepsilon \in\left(0,\left(c_{k}^{\lambda}-a_{k}\right) / 2\right)$ and any $\delta>0$ there exists $u_{\varepsilon} \in E_{\lambda}$ such that $\left|J_{\lambda}\left(u_{\varepsilon}\right)-c_{k}^{\lambda}\right| \leq 2 \varepsilon$ and $\left\|J_{\lambda}^{\prime}\left(u_{\varepsilon}\right)\right\|_{E_{\lambda}^{\prime}} \leq 8 \varepsilon / \delta$. Moreover, it follows from Lemma 4.1 that $\left(c_{k}^{\lambda}-a_{k}\right) / 2 \geq\left(b_{k}^{\lambda}-\right.$ 
$\left.a_{k}\right) / 2 \geq 1 / 2$. Therefore, we can choose $\varepsilon=1 /(4 n)$ and $\delta=8$ so that $\left|J_{\lambda}\left(u_{1 / n}\right)-c_{k}^{\lambda}\right| \leq 1 / n$ and $\left\|J_{\lambda}^{\prime}\left(u_{1 / n}\right)\right\|_{E_{\lambda}^{\prime}} \leq 1 / n$. Then we have a (PS) $c_{k}^{\lambda}$-sequence $\left\{u_{1 / n}\right\}_{n}$.

LEMMA 4.3. For any $(P S)_{c_{k}^{\lambda}}$-sequence $\left\{u_{n}\right\}_{n}$ for $J_{\lambda}$, we have

$$
\limsup _{n \rightarrow \infty}\left\|u_{n}\right\|_{\lambda}^{2} \leq[2 \theta /(\theta-2)] c_{k}^{\lambda}
$$

and $\lim \sup _{\lambda \rightarrow \infty} c_{k}^{\lambda}<\infty$.

Proof. The first inequality follows from Lemma 3.6 (i). Since id $\in \Gamma_{k}^{\lambda}$, we have $c_{k}^{\lambda} \leq \max _{B_{R_{k}}(0) \cap Y_{k}} J_{\lambda}=\max _{B_{R_{k}}(0) \cap Y_{k}} I_{\Omega}$, which is finite and independent of $\lambda$. This shows the finiteness.

LEMMA 4.4. Let $c_{k}^{\lambda}$ be as in (4.2). Set

$$
\underline{c}_{k}^{\lambda}=\sup _{\rho>0} \inf \left\{J_{\lambda}(u) \mid u \in\left(Y_{k-1}^{\lambda}\right)^{\perp} \cap \partial B_{\rho}(0)\right\} .
$$

We have: $c_{k}^{\lambda} \geq \underline{c}_{k}^{\lambda}$ for any large $\lambda$.

PROOF. Let $R_{k}$ be as in Lemma 4.1. If $\rho>0$ satisfies $\left(Y_{k-1}^{\lambda}\right)^{\perp} \cap \partial B_{\rho}(0) \cap Y_{k} \cap$ $\left[\operatorname{int}\left(B_{R_{k}}(0)\right)\right]^{c} \neq \emptyset$, then we deduce from Lemma 4.1 (ii) that

$$
\inf \left\{J_{\lambda}(u) \mid u \in\left(Y_{k-1}^{\lambda}\right)^{\perp} \cap \partial B_{\rho}(0)\right\} \leq 0 \leq c_{k}^{\lambda} .
$$

Next, we consider $\rho>0$ satisfying $\left(Y_{k-1}^{\lambda}\right)^{\perp} \cap \partial B_{\rho}(0) \cap Y_{k} \cap\left[\operatorname{int}\left(B_{R_{k}}(0)\right)\right]^{c}=\emptyset$. For any $\gamma \in \Gamma_{k}^{\lambda}$, we set $\mathcal{O}_{\gamma}=\left\{u \in Y_{k} \cap \operatorname{int}\left(B_{R_{k}}(0)\right) \mid\|\gamma(u)\|_{\lambda}<\rho\right\}$, which is a bounded, symmetric open neighborhood of $0 \in Y_{k}$. Let $P_{k-1}^{\lambda}$ be the orthogonal projection from $E_{\lambda}$ onto $Y_{k-1}^{\lambda}$ for any $k \geq 1$. Since $P_{k-1}^{\lambda} \circ \gamma$ is a continuous odd map from $\overline{\mathcal{O}_{\gamma}}$ to $Y_{k-1}^{\lambda}$, by the Borsuk-Ulam theorem, there exists $v \in \partial \mathcal{O}_{\gamma}$ such that $P_{k-1}^{\lambda}(\gamma(v))=0$, i.e., $\gamma(v) \in\left(Y_{k-1}^{\lambda}\right)^{\perp}$.

Since $v \in \partial \mathcal{O}_{\gamma}$, either (i) $\|v\|_{\lambda}=R_{k}$; or (ii) $\|\gamma(v)\|_{\lambda}=\rho$. If (i) occurs, then $\gamma(v)=$ $v$ since $\gamma \in \Gamma_{k}^{\lambda}$. Since $R_{k}=\|v\|_{\lambda}=\|\gamma(v)\|_{\lambda} \leq \rho$, the element $\left(\rho / R_{k}\right) v$ belongs to $\left(Y_{k-1}^{\lambda}\right)^{\perp} \cap \partial B_{\rho}(0) \cap Y_{k} \cap\left[\operatorname{int}\left(B_{R_{k}}(0)\right)\right]^{c}=\emptyset$. This contradiction shows that $\|v\|_{\lambda}<R_{k}$ and $\|\gamma(v)\|_{\lambda}=\rho$. Therefore, $v \in B_{R_{k}}(0) \cap Y_{k}$ and $\gamma(v) \in\left(Y_{k-1}^{\lambda}\right)^{\perp} \cap \partial B_{\rho}(0)$, and then $\inf \left\{J_{\lambda}(u) \mid u \in\left(Y_{k-1}^{\lambda}\right)^{\perp} \cap \partial B_{\rho}(0)\right\} \leq J_{\lambda}(\gamma(v)) \leq \max _{u \in B_{R_{k}}}(0) \cap Y_{k} J_{\lambda}(\gamma(u))$. This implies that

$$
\inf \left\{J_{\lambda}(u) \mid u \in\left(Y_{k-1}^{\lambda}\right)^{\perp} \cap \partial B_{\rho}(0)\right\} \leq c_{k}^{\lambda} .
$$

The lemma follows from (4.3) and (4.4).

Lemma 4.5. Let $\mu_{k}$ be as in Section 3. There exists $\Lambda_{k}^{\prime}>0$ such that

$$
\underline{c}_{k}^{\lambda} \geq C_{0}\left(\mu_{k}\right)^{\delta}
$$

for any $\lambda \geq \Lambda_{k}^{\prime}$. Here, both $C_{0}>0$ and $\delta>0$ are independent of $\lambda$ and $k$. 
PROOF. By the definition of $\left(Y_{k-1}^{\lambda}\right)^{\perp}$ and Lemma 3.8, we have

$$
\|u\|_{\lambda}^{2} \geq \mu_{k}\|u\|_{L^{2}\left(\mathbf{R}^{N}\right)}^{2} / 2
$$

for any $u \in\left(Y_{k-1}^{\lambda}\right)^{\perp}$ if $\lambda$ is large enough. For $N \geq 3$, it follows from Lemma 3.4, (2.6) with $q=2^{*}$, and (4.5) that

$$
\begin{aligned}
\|u\|_{L^{p}\left(\mathbf{R}^{N}\right)} & \leq\|u\|_{L^{2}\left(\mathbf{R}^{N}\right)}^{a}\|u\|_{L^{2^{*}\left(\mathbf{R}^{N}\right)}}^{1-a} \leq C_{N}\|u\|_{L^{2}\left(\mathbf{R}^{N}\right)}^{a}\|u\|_{\lambda}^{1-a} \\
& \leq C_{N}^{\prime}\|u\|_{\lambda} /\left(\mu_{k}\right)^{a / 2}
\end{aligned}
$$

for any $u \in\left(Y_{k-1}^{\lambda}\right)^{\perp}$. A similar argument replaced $2^{*}$ by $p+1$ yields (4.6) for $N=2$. Here, the exponent $a$ depends only on $p$ and $N$. Then, for any $u \in\left(Y_{k-1}^{\lambda}\right)^{\perp}$ and for any $\varepsilon>0$, we have

$$
\begin{aligned}
J_{\lambda}(u) & \geq \frac{1}{2}\|u\|_{\lambda}^{2}-\left(\varepsilon\|u\|_{L^{2}\left(\mathbf{R}^{N}\right)}^{2}+C_{\varepsilon}\|u\|_{L^{p}\left(\mathbf{R}^{N}\right)}^{p}\right) \\
& \geq \frac{1}{2}\left(1-4 \varepsilon / \mu_{k}\right)\|u\|_{\lambda}^{2}-\frac{C_{\varepsilon}\left(C_{N}^{\prime}\right)^{p}}{\left(\mu_{k}\right)^{a p / 2}}\|u\|_{\lambda}^{p},
\end{aligned}
$$

where we used Lemma 3.1, (4.5), and (4.6) in the second inequality.

Setting $\varepsilon=\mu_{1} / 8$, we have $1-4 \varepsilon / \mu_{k}=1-\mu_{1} /\left(2 \mu_{k}\right) \geq 1 / 2$. We deduce from (4.7) and Lemma 3.3 that

$$
\begin{aligned}
\underline{\mathrm{c}}_{k}^{\lambda} & =\sup _{\rho>0} \inf \left\{J_{\lambda}(u) \mid u \in\left(Y_{k-1}^{\lambda}\right)^{\perp} \cap \partial B_{\rho}(0)\right\} \\
& \geq \sup _{\rho>0}\left(\frac{1}{4} \rho^{2}-\frac{1}{p} \frac{p C_{\varepsilon}\left(C_{N}^{\prime}\right)^{p}}{\left(\mu_{k}\right)^{a p / 2}} \rho^{p}\right) \\
& =\frac{p-2}{2 p}\left(2^{-p} /\left[p C_{\varepsilon}\left(C_{N}^{\prime}\right)^{p} /\left(\mu_{k}\right)^{a p / 2}\right]^{2}\right)^{1 /(p-2)} \\
& =: C_{0}\left(\mu_{k}\right)^{\delta} .
\end{aligned}
$$

This proves the lemma.

LEMmA 4.6. Assume that $B^{-1}(0) \subset B_{R-1}(0)$. Then there exist $C_{k, R}>0$ and $a>0$, both of which are independent of $\lambda$, such that any $(P S)_{c_{k}^{\lambda}}$-sequence $\left\{u_{n}\right\}_{n}$ for $J_{\lambda}$ satisfies $\limsup _{n \rightarrow \infty}\left\|u_{n}\right\|_{L^{p}\left(B_{R}(0)^{c}\right)} \leq C_{k, R} \lambda^{-a}$.

PROOF. By Lemmata 2.4 and 4.3, there exists a constant $C_{k}>0$ such that

$$
\lambda C_{R}\left\|u_{n}\right\|_{L^{2}\left(B_{R}(0)^{c}\right)}^{2} \leq\left\|u_{n}\right\|_{\lambda}^{2} \leq C_{k}
$$

for all $n$ and $\lambda$ large enough. For $N \geq 3$, it follows from Lemma 3.4, (2.6), and (4.8) that

$$
\left\|u_{n}\right\|_{L^{p}\left(B_{R}(0)^{c}\right)} \leq\left\|u_{n}\right\|_{L^{2}\left(B_{R}(0)^{c}\right)}^{a}\left\|u_{n}\right\|_{L^{2^{*}\left(B_{R}(0)^{c}\right)}}^{1-a}
$$




$$
\begin{aligned}
& \leq C_{N}^{1-a}\left\|u_{n}\right\|_{L^{2}\left(B_{R}(0)^{c}\right)}^{a}\left\|u_{n}\right\|_{\lambda}^{1-a} \\
& \leq C_{N}^{1-a}\left(C_{k}\right)^{1 / 2} /\left(C_{R} \lambda\right)^{a / 2},
\end{aligned}
$$

where $a$ is from Lemma 3.4. This completes the proof for $N \geq 3$. A similar argument yields (4.9) for $N=2$.

By Lemmata 4.2 and 3.7, we obtain a critical point $v_{k}^{\lambda} \in E_{\lambda}$ of $J_{\lambda}$. Fix $R>0$ as in Lemma 4.6. It follows from Lemma 3.7 (v) with $v=v_{k}^{\lambda}$ and Lemma 4.6 that

$$
\alpha c_{k}^{\lambda}-C_{k, R} \lambda^{-a} \leq\left\|v_{k}^{\lambda}\right\|_{L^{p}\left(\mathbf{R}^{N}\right)}^{p} \leq C_{p}^{p} C_{k}^{p / 2}=: \beta_{k} .
$$

Here, the constants $\alpha, C_{p}$, and $C_{k}$ are as in Lemma 3.6 (i), (ii), and (4.8), respectively. Thus, by Lemmata 4.5 and 4.4 , for each $k \geq 1$,

$$
\alpha_{k}:=\alpha C_{0}\left(\mu_{k}\right)^{\delta} / 2 \leq \alpha \underline{\mathrm{c}}_{k}^{\lambda} / 2 \leq\left\|v_{k}^{\lambda}\right\|_{L^{p}\left(\mathbf{R}^{N}\right)}^{p} \leq \beta_{k}
$$

for any $\lambda \geq\left[2 C_{k, R} / C_{0}\left(\mu_{k}\right)^{\delta}\right]^{1 / a}+\Lambda_{k}^{\prime}=: \Lambda(k)$. Here, $\Lambda_{k}^{\prime}$ is as in Lemma 4.5.

Since $0<\mu_{1}<\cdots<\mu_{k}<\cdots \rightarrow \infty$ as $k \rightarrow \infty$, it follows from Lemma 4.5 that there exists a sequence $\left\{k_{j}\right\}_{j=1}^{\infty}$ such that $2 \beta_{k_{j}}<\alpha_{k_{j+1}}$ for any $\lambda \geq \Lambda\left(k_{j+1}\right)$. (Clearly, we may assume that $\{\Lambda(k)\}_{k}$ is increasing.)

Therefore, for each $m \geq 1$, the weak solutions $\left\{v_{k_{j}}^{\lambda}\right\}_{j=1}^{m}$ satisfy

$$
\left(0<\alpha_{1}<\right) \alpha_{k_{j}} \leq\left\|v_{k_{j}}^{\lambda}\right\|_{L^{p}\left(\mathbf{R}^{N}\right)}^{p} \leq \beta_{k_{j}}<\alpha_{k_{j+1}} \leq\left\|v_{k_{j+1}}^{\lambda}\right\|_{L^{p}\left(\mathbf{R}^{N}\right)}^{p}
$$

for any $\lambda \geq \Lambda\left(k_{m}\right)$ and for any $j=1,2, \ldots, m-1$.

We now complete the proof of Theorem 1.1 by setting $b_{j}=\left(\beta_{k_{j}}\right)^{1 / p}, a_{j}=\left(\alpha_{k_{j}}\right)^{1 / p}$, and $c_{j}=\sup _{\lambda \geq \Lambda\left(k_{j}\right)}\left\|v_{k_{j}}^{\lambda}\right\|_{\lambda}$, which is finite by Lemma 4.3 and the weak lower-semicontinuity of norms.

\section{Proof of Theorem 1.2}

In this section, we show Theorem 1.2. Our proof is essentially the same as that of Theorem 1.2 in [Ba-Pa-Wa].

Before proceeding to the proof, we recall a standard result from the theory of Sobolev spaces. The next result follows immediately from Corollary 5.3.5 in [Ca] for real-valued functions.

LEMMA 5.1. Let $\Omega$ is a bounded, smooth open subset of $\mathbf{R}^{N}$. Assume that $v \in$ $H^{1}(\Omega), w \in H_{0}^{1}(\Omega)$, and $|v| \leq|w|$ a.e. Then $v \in H_{0}^{1}(\Omega)$.

Let $\left\{\lambda_{n}\right\}_{n=1}^{\infty}$ be a diverging sequence and let $\left\{u_{n}\right\}_{n}$ be a set of critical points of $J_{\lambda_{n}}$ satisfying: $u_{n} \in E_{\lambda_{n}}, \sup _{n}\left\|u_{n}\right\|_{\lambda_{n}}<\infty$, and $\inf _{n}\left\|u_{n}\right\|_{L^{p}\left(\mathbf{R}^{N}\right)}>0$.

We remark that all the statements in Lemma 5.2 below remain valid if the condition " $J_{\lambda_{n}}^{\prime}\left(u_{n}\right)=0$ " is replaced by " $\left\|J_{\lambda_{n}}^{\prime}\left(u_{n}\right)\right\|_{E_{\lambda_{n}}^{\prime}} \rightarrow 0$ as $n \rightarrow \infty$ ". 
LemMA 5.2. There exist $v \in H_{0}^{1}(\Omega)$ and a subsequence of $\left\{u_{n}\right\}_{n}$, still denoted by $\left\{u_{n}\right\}_{n}$, such that $\left.u_{n}\right|_{\Omega} \rightarrow v$ in $H^{1}(\Omega)$, moreover

(i) $u_{n} \rightarrow v$ in $L_{\mathrm{loc}}^{q}\left(\mathbf{R}^{N}\right)$ for any $q \in\left[2,2^{*}\right)$, and $u_{n} \rightarrow v$ in $L^{2}\left(\mathbf{R}^{N}\right)$;

(ii) $u_{n}(x) \rightarrow v(x)$ for a.e. $x \in \mathbf{R}^{N}$;

(iii) $v$ is a weak solution to $-\Delta v=f\left(x,|v|^{2}\right) v$ on $\Omega$.

Proof. By (2.6), we have $\left\|\left|u_{n}\right|\right\|_{H^{1}\left(\mathbf{R}^{N}\right)} \leq C \sup _{n}\left\|u_{n}\right\|_{\lambda_{n}}$, where $C$ is independent of $n$. Thus, $\left\{\left|u_{n}\right|\right\}_{n}$ is bounded in $H^{1}\left(\mathbf{R}^{N}\right)$, therefore we find a (renamed) subsequence such that $\left|u_{n}\right| \rightarrow w \in H^{1}\left(\mathbf{R}^{N}\right) ;\left|u_{n}\right| \rightarrow w$ in $L_{\text {loc }}^{q}\left(\mathbf{R}^{N}\right)$ for any $q \in\left[2,2^{*}\right) ;\left|u_{n}(x)\right| \rightarrow w(x)$ for a.e. $x \in \mathbf{R}^{N}$ as $n \rightarrow \infty$.

Let $\delta>0$. It follows from Corollary 2.6 that $\lambda_{n}\left\|u_{n}\right\|_{L^{2}\left(\mathbf{R}^{N} \backslash\left[B^{-1}(0)\right]_{\delta}^{+}\right)}^{2} \leq C_{\delta} \sup _{n}\left\|u_{n}\right\|_{\lambda_{n}}^{2}$, where $C_{\delta}$ is independent of $n$. Thus,

$$
\lim _{n \rightarrow \infty}\left\|u_{n}\right\|_{L^{2}\left(\mathbf{R}^{N} \backslash\left[B^{-1}(0)\right]_{\delta}^{+}\right)}^{2}=0
$$

since $\lambda_{n} \rightarrow \infty$. Using a countable covering argument, we choose a (renamed) subsequence such that $u_{n}(x) \rightarrow 0$ for a.e. $x \in\left[B^{-1}(0)\right]^{c}$. Therefore, $w(x)=0$ for a.e. $x \in \Omega^{c}$ because of (A.5). Since $\partial \Omega$ has zero measure, we conclude that $w \in H_{0}^{1}(\Omega)$ (e.g., [Br], Theorem 9.18).

On the other hand, $\left\|\nabla u_{n}\right\|_{L^{2}\left(\Omega, \mathbf{C}^{N}\right)} \leq\left\|\nabla_{\lambda A} u_{n}\right\|_{L^{2}\left(\mathbf{R}^{N}, \mathbf{C}^{N}\right)} \leq \sup _{n}\left\|u_{n}\right\|_{\lambda_{n}}$ by (A.5). Thus, $\left\{\left.u_{n}\right|_{\Omega}\right\}_{n}$ is bounded in $H^{1}(\Omega)$, and then we find a subsequence, still denoted by $\left\{u_{n}\right\}_{n}$, such that $\left.u_{n}\right|_{\Omega} \rightarrow v \in H^{1}(\Omega) ;\left.u_{n}\right|_{\Omega} \rightarrow v$ in $L^{q}(\Omega)$ for any $q \in\left[2,2^{*}\right) ; u_{n}(x) \rightarrow v(x)$ for a.e. $x \in \Omega$. Hence, $|v(x)|=w(x)$ for a.e. $x \in \Omega$, and then $v \in H_{0}^{1}(\Omega)$ by Lemma 5.1.

Let $q \in\left[2,2^{*}\right)$. For any bounded measurable set $K$, we have $\left\|u_{n}-v\right\|_{L^{q}(K)}^{q}=$ $\left\|\left|u_{n}\right|\right\|_{L^{q}(K \backslash \Omega)}^{q}+\left\|\left.u_{n}\right|_{\Omega}-v\right\|_{L^{q}(K \cap \Omega)}^{q} \rightarrow 0$ as $n \rightarrow \infty$ by the $L_{\text {loc }}^{q}$-convergences. Moreover, by (5.1) and the $L_{\text {loc }}^{2}$-convergence, we have

$$
\left\|u_{n}-v\right\|_{L^{2}\left(\mathbf{R}^{N}\right)}^{2}=\left\|u_{n}\right\|_{L^{2}\left(\mathbf{R}^{N} \backslash\left[B^{-1}(0)\right]_{\delta}^{+}\right)}^{2}+\left\|u_{n}-v\right\|_{L^{2}\left(\left[B^{-1}(0)\right]_{\delta}^{+}\right)}^{2} \rightarrow 0
$$

as $n \rightarrow \infty$. These imply (i) and (ii).

Finally, We show (iii). Every $u_{n}$ satisfies $E_{\lambda_{n}}^{\prime}\left\langle J_{\lambda_{n}}^{\prime}\left(u_{n}\right), \varphi\right\rangle_{E_{\lambda_{n}}}=0$ for any $\varphi \in H_{0}^{1}(\Omega)$. Recall that $\nabla\left(\left.u_{n}\right|_{\Omega}\right)=\left.\left(\nabla u_{n}\right)\right|_{\Omega} ;\left.u_{n}\right|_{\Omega} \rightarrow v$ in $H^{1}(\Omega)$; and $\left.u_{n}\right|_{\Omega} \rightarrow v$ in $L_{\text {loc }}^{q}(\Omega)$. Then we can deduce from Lemma 3.2 that $v$ is a weak solution to (1.6).

We recall a lemma due to Lions (Lemma I.1 in [Li]):

LEMmA 5.3. Let $r>0$ and $q^{\prime} \in\left[2,2^{*}\right)$. Assume that $\left\{g_{n}\right\}_{n}$ is bounded in $H^{1}\left(\mathbf{R}^{N}\right)$ and satisfies $\lim _{n \rightarrow \infty} \sup _{y \in \mathbf{R}^{N}} \int_{B_{r}(y)}\left|g_{n}(x)\right|^{q^{\prime}} d x=0$. Then $\left\{g_{n}\right\}_{n}$ converges to 0 in $L^{q}\left(\mathbf{R}^{N}\right)$ for any $q \in\left(2,2^{*}\right)$.

LEMMA 5.4. Let $\left\{u_{n}\right\}_{n}$ and $v$ be as in lemma 5.2. A renamed subsequence satisfies: $u_{n} \rightarrow v$ as $n \rightarrow \infty$ in $L^{q}\left(\mathbf{R}^{N}\right)$ for any $q \in\left(2,2^{*}\right)$. Moreover, $v \neq 0$. 
PROOF. Set $g_{n}=u_{n}-v\left(\in E_{\lambda_{n}}\right)$. It follows from (2.6) that $\left\|\left|g_{n}\right|\right\|_{H^{1}\left(\mathbf{R}^{N}\right)} \leq C\left\|g_{n}\right\|_{\lambda_{n}} \leq$ $C\left(\sup _{n}\left\|u_{n}\right\|_{\lambda_{n}}+\|v\|_{H^{1}(\Omega)}\right)<\infty$. Thus, $\left\{\left|g_{n}\right|\right\}_{n}$ is bounded in $H^{1}\left(\mathbf{R}^{N}\right)$ and $g_{n}(x) \rightarrow 0$ for a.e. $x \in \mathbf{R}^{N}$ by Lemma 5.2 (ii).

We show that $\left|g_{n}\right| \rightarrow 0$ in $L^{q}\left(\mathbf{R}^{N}\right)$ for any $q \in\left(2,2^{*}\right)$ by contradiction. Assume that $\left\{\left|g_{n}\right|\right\}_{n}$ does not converges to 0 in $L^{q}\left(\mathbf{R}^{N}\right)$ for some $q \in\left(2,2^{*}\right)$. Then, by Lemma 5.3 with $r=1, q^{\prime}=2$, and $g_{n}=\left|g_{n}\right|$, there exist $\delta>0$, a sequence $\left\{y_{n}\right\}_{n} \subset \mathbf{R}^{N}$, and a (renamed) subsequence $\left\{\left|g_{n}\right|\right\}_{n}$ such that

$$
\inf _{n}\left\|g_{n}\right\|_{L^{2}\left(B_{1}\left(y_{n}\right)\right)}^{2} \geq \delta .
$$

Moreover, we have $\left|y_{n}\right| \rightarrow \infty$ since $g_{n} \rightarrow 0$ in $L_{\text {loc }}^{2}\left(\mathbf{R}^{N}\right)$ by Lemma 5.2 (i), and we have $g_{n}=u_{n}-v=u_{n}$ on $B_{1}\left(y_{n}\right)$ for any large $n$ since $v \in H_{0}^{1}(\Omega)$. Then it follows from (5.2) and Corollary 2.5 that

$$
\begin{aligned}
\lambda_{n} \delta & \leq \lambda_{n}\left\|g_{n}\right\|_{L^{2}\left(B_{1}\left(y_{n}\right)\right)}^{2}=\lambda_{n}\left\|u_{n}\right\|_{L^{2}\left(B_{1}\left(y_{n}\right)\right)}^{2} \\
& \leq C^{\prime}\left\|u_{n}\right\|_{\lambda_{n}}^{2} \leq C^{\prime} \sup _{n}\left\|u_{n}\right\|_{\lambda_{n}}^{2}<\infty,
\end{aligned}
$$

where $C^{\prime}$ is independent of $n$. Since $\lambda_{n} \rightarrow \infty$, this is a contradiction.

Finally, we have $\|v\|_{L^{p}\left(\mathbf{R}^{N}\right)} \geq \inf _{n}\left\|u_{n}\right\|_{L^{p}\left(\mathbf{R}^{N}\right)}-\limsup _{n \rightarrow \infty}\left\|u_{n}-v\right\|_{L^{p}\left(\mathbf{R}^{N}\right)}=$ $\inf _{n}\left\|u_{n}\right\|_{L^{p}\left(\mathbf{R}^{N}\right)}>0$ by assumption. This shows that $v \neq 0$.

LEMMA 5.5. Let $\left\{u_{n}\right\}_{n}$ and $v$ be as in lemma 5.4. A renamed subsequence satisfies: (i) $\left\|u_{n}-v\right\|_{\lambda_{n}} \rightarrow 0$, and (ii) $J_{\lambda_{n}}\left(u_{n}\right) \rightarrow I_{\Omega}(v)$, as $\lambda \rightarrow \infty$.

Proof. We show (i). Since $v \in H_{0}^{1}(\Omega)$, it follows from (A.5) that

$$
\begin{aligned}
& E_{\lambda_{n}}^{\prime}\left\langle J_{\lambda_{n}}^{\prime}(v), u_{n}-v\right\rangle_{E_{\lambda_{n}}} \\
& \quad=\operatorname{Re}\left(\int_{\Omega} \nabla v \overline{\nabla\left(u_{n}-v\right)} d x+\int_{\Omega} f\left(x,|v|^{2}\right) v \overline{\left(u_{n}-v\right)} d x\right) .
\end{aligned}
$$

The first term on the right-hand side of (5.3) tends to 0 as $n \rightarrow \infty$ since $\left.u_{n}\right|_{\Omega} \rightarrow v$ in $H^{1}(\Omega)$ by Lemma 5.2. By the $L_{\text {loc }}^{q}$-convergence of $\left\{u_{n}\right\}_{n}$ and Lemma 3.1 (iii), the second integral on the right-hand side of (5.3) tends to 0 as $n \rightarrow \infty$. Thus, we have $\lim _{n \rightarrow \infty}\left\langle J_{\lambda_{n}}^{\prime}(v), u_{n}-v\right\rangle=0$. Since $J_{\lambda_{n}}^{\prime}\left(u_{n}\right)=0$, it follows that, as $n \rightarrow \infty$,

$$
\begin{aligned}
o(1)= & E_{\lambda_{n}}\left\langle J_{\lambda_{n}}^{\prime}\left(u_{n}\right)-J_{\lambda_{n}}^{\prime}(v), u_{n}-v\right\rangle_{{\lambda_{n}}_{n}} \\
= & \left\|u_{n}-v\right\|_{\lambda_{n}}^{2} \\
& -\operatorname{Re} \int_{\mathbf{R}^{N}} f\left(\left|u_{n}\right|^{2}\right) u_{n} \overline{\left(u_{n}-v\right)} d x+\operatorname{Re} \int_{\Omega} f\left(|v|^{2}\right) v \overline{\left(u_{n}-v\right)} d x .
\end{aligned}
$$


From this, using Lemma 3.1 (iii) with $\varepsilon=1$ and the $L_{\text {loc }}^{q}$-convergence, we have

$$
\begin{aligned}
& \left\|u_{n}-v\right\|_{\lambda_{n}}^{2} \\
& \quad \leq\left(\|v\|_{L^{2}(\Omega)}\left\|u_{n}-v\right\|_{L^{2}(\Omega)}+C_{1}\|v\|_{L^{p}(\Omega)}^{p-1}\left\|u_{n}-v\right\|_{L^{2}(\Omega)}\right)+o(1) \\
& \quad=\left(\left\|u_{n}\right\|_{L^{2}\left(\mathbf{R}^{N}\right)}\left\|u_{n}-v\right\|_{L^{2}\left(\mathbf{R}^{N}\right)}+C_{1}\left\|u_{n}\right\|_{L^{p}\left(\mathbf{R}^{N}\right)}^{p-1}\left\|u_{n}-v\right\|_{L^{p}\left(\mathbf{R}^{N}\right)}\right)+o(1) \\
& \quad \leq C_{p}\left(\sup _{n}\left\|u_{n}\right\|_{\lambda_{n}}+\sup _{n}\left\|u_{n}\right\|_{\lambda_{n}}^{p-1}\right)\left(\left\|u_{n}-v\right\|_{L^{2}\left(\mathbf{R}^{N}\right)}+\left\|u_{n}-v\right\|_{L^{p}\left(\mathbf{R}^{N}\right)}\right)+o(1) \\
& \quad \leq C_{p}^{\prime}\left(\left\|u_{n}-v\right\|_{L^{2}\left(\mathbf{R}^{N}\right)}+\left\|u_{n}-v\right\|_{L^{p}\left(\mathbf{R}^{N}\right)}\right)+o(1)
\end{aligned}
$$

as $n \rightarrow \infty$, where we used (2.6) in the first inequality and used the finiteness assumption. Then the assertion (i) follows from Lemmata 5.4 and 5.2 (i).

The assertion (ii) follows from (i) and the $L^{p}\left(\mathbf{R}^{N}\right)$-convergence of $u_{n}$ to $v$ from Lemma 5.4. Indeed, since $v \in H_{0}^{1}(\Omega)$, we have

$$
J_{\lambda_{n}}\left(u_{n}\right)-I_{\Omega}(v)=\frac{1}{2}\left(\left\|u_{n}\right\|_{\lambda_{n}}^{2}-\|v\|_{\lambda_{n}}^{2}\right)-\int_{\mathbf{R}^{N}}\left(F\left(x,\left|u_{n}\right|^{2}\right)-F\left(x,|v|^{2}\right)\right) d x .
$$

The first term on the right-hand side tends to zero as $n \rightarrow \infty$ by (i). The second term tends to zero by Lemma 3.1 (iv).

We now complete the proof of Theorem 1.2.

\section{Proof of Theorem 1.3}

In this section, we show Theorem 1.3. Assume (A.1)-(A.5) and (f.1)-(f.6). We may assume that $\lambda$ is large enough.

Under the assumptions (f.1)-(f.5), we can show the positivity of the infimum $c_{\lambda}$ over the Nehari manifold and the existence of a $(\mathrm{PS})_{c_{\lambda}}$-sequence for $J_{\lambda}$; this can be shown as in the proof of Theorem 4.2 in [Wi]. Moreover, we have $\liminf _{\lambda \rightarrow \infty} c_{\lambda} \geq b>0$, where $b$ is as in Lemma 3.5, because of the mountain pass type characterization of $c_{\lambda}$ obtained as in Section 4 in [Wi].

In addition, under (f.6), we can show that $J_{\lambda}$ satisfies the (PS) $c_{\lambda}$-condition for any large $\lambda$ by using the results in Subsection 3.1 and the argument in the proof of Lemma 4.6; the proof is similar to that of Proposition 2.1 in [Ba-Wa2]. Therefore, the value $c_{\lambda}$ is attained by some critical point $u_{\lambda} \in E_{\lambda}$ for $J_{\lambda}$ (e.g., [Wi]). Note that it follows from the definition of the Nehari manifolds that $(0<b \leq) c_{\lambda} \leq c(\Omega)$ since $H_{0}^{1}(\Omega) \subset E_{\lambda}$.

Finally, we show that $c_{\lambda} \rightarrow c(\Omega)$ as $\lambda \rightarrow \infty$ by contradiction. Assume that there exists a diverging sequence $\left\{\lambda_{n}\right\}_{n}$ such that $c_{\lambda_{n}}=J_{\lambda_{n}}\left(u_{\lambda_{n}}\right) \rightarrow A \in(0, c(\Omega))$ as $n \rightarrow \infty$. Then the sequence $\left\{u_{\lambda_{n}}\right\}_{n}$ satisfies the conditions: $J_{\lambda_{n}}^{\prime}\left(u_{\lambda_{n}}\right)=0, \sup _{n}\left\|u_{\lambda_{n}}\right\|_{\lambda_{n}}<\infty$, and $\inf _{n}\left\|u_{\lambda_{n}}\right\|_{L^{p}\left(\mathbf{R}^{N}\right)}>0$ by Lemma 3.6 (i), (iv). In particular, all the assumptions of Theorem 1.2 with $u_{n}=u_{\lambda_{n}}$ are fulfilled. Hence, we find a (renamed) subsequence such that $J_{\lambda_{n}}\left(u_{\lambda_{n}}\right) \rightarrow$ 
$I_{\Omega}(v)$ for some nonzero critical point $v$ of $I_{\Omega}$. Therefore, $c(\Omega) \leq I_{\Omega}(v)=A<c(\Omega)$. This contradiction finishes the proof.

\section{References}

[Al-So] C. O. Alves and M. A. S. Souto, Multiplicity of positive solutions for a class of problems with critical growth in $\mathbf{R}^{2}$, J. Differential Equations 244 (2008), No. 6, 1502-1520.

[Al-Mo-So] C. O. Alves, D. C. De Morais Filho and M. A. Souto, Multiplicity of positive solutions for a class of problems with critical growth in $\mathbf{R}^{N}$, Proc. Edinb. Math. Soc. (2) 52 (2009), 1-21.

[Al] C. O. Alves, Multiplicity of multi-bump type nodal solutions for a class of elliptic problems in $\mathbf{R}^{N}$, Topol. Methods in Nonlinear Anal. 34 (2009), No. 2, 231-250.

[Av-He-Si] J. E. Avron, I. Herbst and B. Simon, Schrödinger operators with magnetic fields. I. General interactions, Duke Math. J. 45 (1978), no. 4, 847-883.

[Br] H. BREZIS, Functional Analysis, Sobolev spaces and Partial Differential Equations, Springer-Verlag, Universitext (2010).

[Ba-Pa-Wa] T. BARtsch, A. PAnkov and Z.-Q. WAng, Nonlinear Schrödinger equations with steep potential well, Commun. Contemp. Math. 3 (2001), no. 4, 549-569.

[Ba-Wa] T. BARTSCH and Z.-Q. WANG, Existence and multiplicity results for some superlinear elliptic problems on $\mathbf{R}^{N}$, Comm. Partial Differential Equations 20 (1995), no. 9-10, 1725-1741.

[Ba-Wa2] T. BARTSCH and Z.-Q. WANG, Multiple positive solutions for a nonlinear Schrödinger equation, Z. Angew. Math. Phys. 51 (2000), 366-384.

[Ca] T. CAZEnave, An Introduction to semilinear elliptic equations, Editora do IM-UFRJ, Rio de Janeiro (2006).

[Cl-Di] M. ClapP and Y. DING, Positive solutions of a Schrödinger equation with critical nonlinearity, Z. Angew. Math. Phys. 55 (2004), no. 4, 592-605.

[De-Di] D. G. De Figueiredo and Y. Ding, Solutions of a nonlinear Schrödinger equation, Discrete Contin. Dyn. Syst. 8 (2002), no. 3, 563-584.

[Di-Ta] Y. Ding and K. TANAKA, Multiplicity of positive solutions of a nonlinear Schrödinger equation, Manuscripta Math. 112 (2003), no. 1, 109-135.

[He-He] R. Hempel and I. Herbst, Strong magnetic fields, Dirichlet boundaries, and spectral gaps, Comm. Math. Phys. 169 (1995), 237-259.

[He-Na] I. HERBST and S. NAKAMURA, Schrödinger operators with strong magnetic fields: quasi-periodicity of spectral orbits and topology, Differential operators and spectral theory, 105-123, Amer. Math. Soc. Transl. Ser. 2, 189, Amer. Math. Soc., Providence, RI, 1999.

[Le-Si] H. LEINFELDER and C. G. SIMADER, Schrödinger operators with singular magnetic vector potentials, Math. Z. 176 (1981), no. 1, 1-19.

[Li] P. L. Lions, The concentration-compactness principle in the calculus of variations. The locally compact case. II, Ann. Inst. H. Poincaré Anal. Non Linéaire 1 (1984), no. 4, 223-283.

[Re-Si] M. ReED and B. Simon, Methods of Modern Mathematical Physics, Vol. I, Academic Press, New York (1980).

[Zo-Sc] M. Schechter and W. Zou, Critical point theory and its applications, Springer, New York (2006).

[Si] B. SIRAKOV, Existence and multiplicity of solutions of semilinear elliptic equations in $\mathbf{R}^{N}$, Calc. Var. 11 (2000), 119-142.

[Ta1] Z. TANG, On the least energy solutions of nonlinear Schrödinger equations with electromagnetic fields, Comput. Math. Appl. 54 (2007), no. 5, 627-637.

[Ta2] Z. TANG, Multiplicity of standing wave solutions of nonlinear Schrödinger equations with electromagnetic fields, Z. Angew. Math. Phys. 59 (2008), no. 5, 810-833. 
[Ta3] Z. TANG, Multi-bump bound states of nonlinear Schrödinger equations with electromagnetic fields and critical frequency, J. Differential Equations 245 (2008), no. 10, 2723-2748.

[Wi] M. Willem, Minimax theorems, Birkhäuser, Boston (1996).

Present Address:

DEPARTMENT OF MATHEMATICS,

OSAKA INSTITUTE OF TECHNOLOGY,

ASAHI, OSAKA, 535-8585 JAPAN.

e-mail: shirai@ge.oit.ac.jp 